\title{
Unified chiral analysis of the vector meson spectrum from lattice QCD
}

\author{
W Armour ${ }^{1}$, C R Allton ${ }^{1}$, D B Leinweber ${ }^{2,3}$, A W Thomas 4 \\ and R D Young ${ }^{4}$ \\ ${ }^{1}$ Department of Physics, University of Wales Swansea, Swansea, SA2 8PP, Wales, UK \\ ${ }^{2}$ Special Research Center for the Subatomic Structure of Matter, University of Adelaide, \\ Adelaide, SA 5005, Australia \\ ${ }^{3}$ Department of Physics, University of Adelaide, Adelaide, SA 5005, Australia \\ ${ }^{4}$ Jefferson Lab, 12000 Jefferson Ave., Newport News, VA 23606, USA
}

Received 21 October 2005

Published 26 May 2006

Online at stacks.iop.org/JPhysG/32/971

\begin{abstract}
The chiral extrapolation of the vector meson mass calculated in partially quenched lattice simulations is investigated. The leading one-loop corrections to the vector meson mass are derived for partially quenched QCD. A large sample of lattice results from the CP-PACS Collaboration is analysed, with explicit corrections for finite lattice spacing artefacts. To incorporate the effect of the opening decay channel as the chiral limit is approached, the extrapolation is studied using a necessary phenomenological extension of chiral effective field theory. This chiral analysis also provides a quantitative estimate of the leading finite volume corrections. It is found that the discretization, finitevolume and partial quenching effects can all be very well described in this framework, producing an extrapolated value of $M_{\rho}$ in excellent agreement with experiment. This procedure is also compared with extrapolations based on polynomial forms, where the results are much less enlightening.
\end{abstract}

(Some figures in this article are in colour only in the electronic version)

\section{Introduction}

There has been great progress in lattice QCD in recent years, associated both with Moore's Law and with improved algorithms, which mean that one can work with larger lattice spacings and still approximate the continuum limit well. The CP-PACS group has devoted considerable effort to the study of the masses of the lowest mass baryons and vector mesons. This has led, for example, to a comprehensive set of data for the mass of the $\rho$-meson in partially quenched QCD, with exceptionally small statistical errors [1]. We shall exploit these data.

The remaining barrier to direct comparison with experimental data is the fact that calculations take much longer as the quark-mass approaches the chiral limit. Indeed the 
time for a given calculation scales somewhere in the range $m_{\pi}^{-7}$ to $m_{\pi}^{-9}$, depending on how hard one works to preserve chiral symmetry. As a result, there has been considerable interest in using chiral perturbation theory ( $\chi$ PT), an effective field theory (EFT) built on the symmetries of QCD, to provide a functional form for hadron properties as a function of quark mass [2, 3]. In principle, such a functional form can then be used to extrapolate from the large pion masses where lattice data exist to the physical value. Unfortunately, there is considerable evidence that the convergence of dimensionally regularized $\chi \mathrm{PT}$ is too slow for this expansion to be reliable at present [4-10].

On the other hand, it can be shown that a reformulation of $\chi \mathrm{PT}$ using finite-range regularization (FRR) effectively resums the chiral expansion, leaving a residual series with much better convergence properties $[2,7]$. The FRR expansion is mathematically equivalent to dimensionally regularized $\chi \mathrm{PT}$ to the finite order one is working $[7,11]$. Systematic errors associated with the functional form of the regulator are at the fraction of a per cent level [2]. A formal description of the formulation of baryon $\chi \mathrm{PT}$ using a momentum cutoff (or FRR) has recently been considered by Djukanovic et al [12]. The price of such an approach is a residual dependence on the regulator mass, which governs the manner in which the loop integrals vanish as the pion mass grows large. However, if it can be demonstrated that reasonable variation of this mass does not significantly change the extrapolated values of physical properties, one has made progress. This seems to be the case for the nucleon mass [9] and magnetic moments [13], for example, where 'reasonable variation' is taken to be $\pm 20 \%$ around the best fit value of the optimal regulator mass.

In order to test whether the problem is indeed solved in this way one needs a large body of accurate data. This is in fact available for the $\rho$-meson, where CP-PACS has carried out lattice simulations of partially quenched QCD (pQQCD) with a wide range of sea and valence masses. This sector requires a modified effective field theory, namely partially quenched chiral perturbation theory $(\mathrm{PQ} \chi \mathrm{PT})[14,15]$. Formal developments in this field have made significant progress in the study of a range of hadronic observables—see [16-22] for example.

In considering the $\rho$-meson, analysis of modern lattice results requires one to extend beyond the low-energy EFT. Near the chiral limit, the $\rho$ decays to two energetic pions, whereas at the quark masses simulated on the lattice the $\rho$ is stable. The pions contributing to the imaginary part of the $\rho$-mass cannot be considered soft, and therefore cannot be systematically incorporated into a low-energy counting scheme [23, 24]. Because almost all the lattice simulation points in this analysis lie in the region $m_{\pi}>m_{\rho} / 2$, it is evident that the extrapolation to the chiral regime will encounter a threshold effect where the decay channel opens. To incorporate this physical threshold, we model the $\rho \rightarrow \pi \pi$ self-energy diagram constrained to reproduce the observed width at the physical pion mass. Including this contribution also provides a model of the finite volume corrections arising from the infrared component of the loop integral. In particular, we can also describe the lattice results in the region $m_{\pi}<m_{\rho} / 2$, where the decay channel is still energetically forbidden because of momentum discretization.

This large body of pQQCD simulation data is analysed within a framework which incorporates the leading low-energy behaviour of partially quenched EFT, together with a model for describing the decay channel of the $\rho$-meson. Finite-range regularization is implemented to evaluate loop integrals, for reasons discussed above. The aim is to test whether it produces a more satisfactory description of the complete data set than the more commonly used, naive extrapolation formulae. A condensed version of some the work featured here has been reported in [25].

The next section summarizes the finite-range regularized forms for the self-energy of the $\rho$-meson in the case of pQQCD. Section 3 discusses the data used from the CP-PACS 


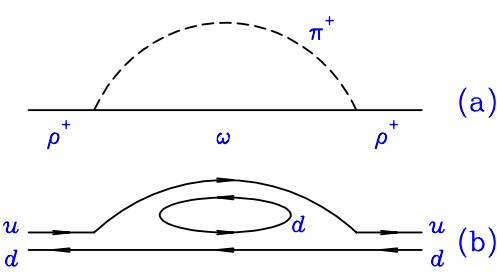

Figure 1. The leading non-analytic contribution to the chiral expansion of the $\rho$-meson mass. The meson dressing (a) and its associated quark-flow diagram (b) are illustrated.

Collaboration [1]. We then give details of the chiral fits in section 4. Finally, section 5 reports the experimental determination of the $\rho$-meson mass at the physical point.

\section{Self-energies for the partially quenched analysis}

Theoretical calculations of dynamical-fermion QCD provide an opportunity to explore the properties of QCD in an expansive manner. The idea is that the sea-quark masses (considered in creating the gauge fields of the QCD vacuum) and valence-quark masses (associated with operators acting on the QCD vacuum) need not match. Such simulation results are commonly referred to as partially quenched calculations. Unlike quenched QCD, which connects to full QCD only in the heavy quark limit, partially quenched QCD is not an approximation. The chiral coefficients of terms in the chiral expansion (such as the axial couplings of the $\pi$ and $\left.\eta^{\prime}\right)$ are the same as in full QCD. Hence, the results of partially quenched QCD provide a theoretical extension of QCD [15]. QCD, as realized in nature, is recovered in the limit where the valence and sea masses match.

In this section we explain the form of the finite-range regularized chiral extrapolation formula in the case of partially quenched QCD (pQQCD) - i.e., the case where the valence and sea quarks are not necessarily mass degenerate. This work extends on the early work of [26] and the more recent analysis of [27], which studied the case of physical (full) QCD. This discussion includes the self-energies $\Sigma_{\pi \pi}^{\rho}$ and $\Sigma_{\pi \omega}^{\rho}$ (corresponding to equations (3) and (4) in [27]) and includes the self-energy contributions associated with the double hairpin diagrams surviving to some extent in pQQCD. We restrict our attention to the case where the two valence quarks in the vector meson are degenerate.

We introduce the following notation. $M_{\mathrm{PS}(V)}\left(\beta, \kappa_{\mathrm{sea}} ; \kappa_{\mathrm{val}}^{1}, \kappa_{\mathrm{val}}^{2}\right)$ refers to the pseudoscalar (vector) meson mass, with the first two arguments referring to the gauge coupling and seaquark mass, while the last two refer to the valence-quark masses. Throughout the paper it will be convenient to use a short-hand notation,

$$
\begin{aligned}
& M^{\text {non-deg }}=M\left(\beta, \kappa_{\text {sea }} ; \kappa_{\text {sea }}, \kappa_{\text {val }}\right) \\
& M^{\text {deg }}=M\left(\beta, \kappa_{\text {sea }} ; \kappa_{\text {val }}, \kappa_{\text {val }}\right) \\
& M^{\text {unit }}=M\left(\beta, \kappa_{\text {sea }} ; \kappa_{\text {sea }}, \kappa_{\text {sea }}\right),
\end{aligned}
$$

where the superscript 'unit' refers to the unitary data with $\kappa_{\mathrm{val}}^{1} \equiv \kappa_{\mathrm{val}}^{2} \equiv \kappa_{\text {sea }}$; 'deg' refers to the 'degenerate' data, where $\kappa_{\text {val }}^{1} \equiv \kappa_{\text {val }}^{2}$ and these are not necessarily equal to $\kappa_{\text {sea }}$; 'non-deg' refers to the non-degenerate case where $\kappa_{\text {val }}^{1}=\kappa_{\text {sea }} \neq \kappa_{\text {val }}^{2}$.

Derivation of the pQQCD chiral expansion for mesons can be described via the diagrammatic method [18], where the role of sea-quark loops in the creation of pseudoscalar meson dressings of the vector meson is easily observed. Consider the simplest case of the $\pi-\omega$ dressing of the $\rho$-meson depicted in figure 1 , which gives rise to the leading non-analytic 


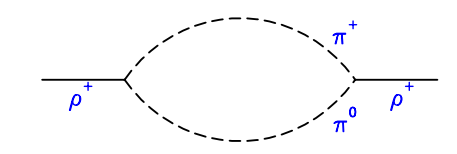

(a)
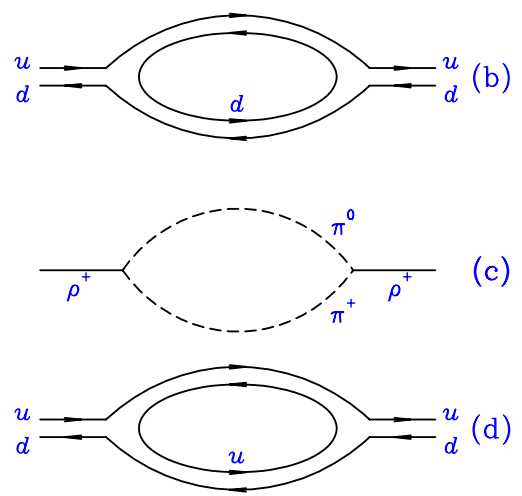

Figure 2. Two-pion contributions to the positively charged $\rho$-meson self-energy. The quarkflow diagrams corresponding to the meson dressings of (a) and (c) are illustrated in (b) and (d), respectively.

(LNA), $m_{\pi}^{3}$, contribution to the chiral expansion of the $\rho$-meson mass in full QCD. Here the positive charge state of the $\rho$ is selected to simplify the derivation.

The two-pion contribution to the $\rho$-meson self-energy is depicted in figure 2 . This channel gives rise to the next-to-leading non-analytic (NLNA), $m_{\pi}^{4} \log m_{\pi}$, contribution to the chiral expansion of the $\rho$-meson mass. Importantly, this contribution also ensures that the $\rho$ develops a finite width as the two- $\pi$ decay becomes accessible.

As these channels can only come about through the inclusion of a sea-quark loop, the expressions for the pionic self-energies are as given in [27], but with the pion mass being $M_{\mathrm{PS}}^{\text {non-deg }}$, corresponding to one valence quark and one sea quark.

In the case of the partially quenched $\eta^{\prime}$ contributions to the chiral expansion of pQQCD, we simplify the calculation by assuming (as in [28]) that the Witten-Veneziano coupling is simply a constant, $-\mu_{0}^{2}$, and take the limit $\mu_{0} \rightarrow \infty$ after summing all relevant sea quark bubble diagrams. The philosophy is that the physical $\eta^{\prime}$ mass is so large that we can ignore $\eta^{\prime}$ loops in full QCD.

The $\eta^{\prime}$ contributions to the $\rho$-meson self-energy are depicted in figure 3(a) with the associated quark-flow diagrams illustrated in figures 3(b) through (f). While figure 3(c) appears in quenched QCD, it is complemented by an infinite series of terms, the first few of which are depicted in figures 3(d) through (f). Only the sum of figures 3(b) through (f) and beyond raise the $\eta^{\prime}$ mass in full QCD.

In partially quenched QCD it is essential to track the masses of the pseudoscalar mesons contributing to the quark-flow diagrams of figure 3. Whereas figure 3(b) involves a nondegenerate pseudoscalar meson, figure 3(c) involves degenerate pseudoscalar mesons, and the remaining figures involve both degenerate and non-degenerate pseudoscalar mesons. The sum of diagrams (b) through (f) of figure 3 and beyond generate an $\eta^{\prime}$ propagator proportional to

$\frac{g_{2}^{2}}{q^{2}+M_{\mathrm{PS}}^{\text {non-deg }}{ }^{2}}-\frac{g_{2}^{2} \mu_{0}^{2}}{\left(q^{2}+M_{\mathrm{PS}}^{\mathrm{deg}^{2}}\right)^{2}}\left[1-\frac{\mu_{0}^{2}}{q^{2}+M_{\mathrm{PS}}^{\mathrm{uni}^{2}}}+\left(\frac{\mu_{0}^{2}}{q^{2}+M_{\mathrm{PS}}^{\mathrm{uni}^{2}}}\right)^{2}-\cdots\right]$. 


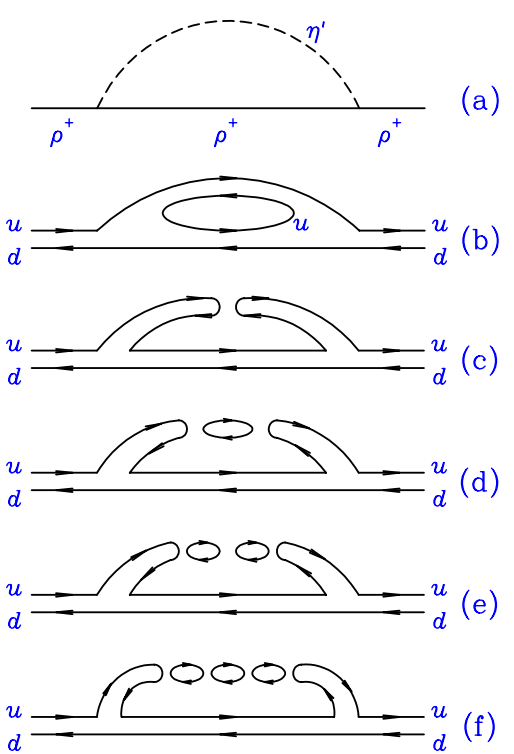

Figure 3. The $\eta^{\prime}$ contribution to the $\rho$-meson self-energy and its associated quark-flow diagrams in PQQCD. While diagram (c) appears in quenched QCD, it is complemented by an infinite series of terms, the first few of which are depicted in diagrams (d) through (f).

Upon summing the terms in $[\cdots]$, expression (1) takes the form

$$
\frac{g_{2}^{2}}{q^{2}+M_{\mathrm{PS}}^{\text {non- } \mathrm{deg}^{2}}}-g_{2}^{2} \frac{q^{2}+M_{\mathrm{PS}}^{\mathrm{unit}^{2}}}{\left(q^{2}+M_{\mathrm{PS}}^{\mathrm{deg}^{2}}\right)^{2}} \frac{\mu_{0}^{2}}{q^{2}+M_{\mathrm{PS}}^{\mathrm{unit}^{2}}+\mu_{0}^{2}} .
$$

We note that for equal valence- and sea-quark masses where $M_{\mathrm{PS}}^{\mathrm{deg}}=M_{\mathrm{PS}}^{\text {non-deg }}=M_{\mathrm{PS}}^{\text {unit }}$, expression (2) describes the propagation of a heavy $\eta^{\prime}$ meson. Upon taking $\mu_{0} \rightarrow \infty$

$$
\sigma_{\eta^{\prime} \rho}^{\mathrm{tot}} \sim \frac{g_{2}^{2}}{q^{2}+M_{\mathrm{PS}}^{\mathrm{non}-\mathrm{deg}^{2}}}-\frac{g_{2}^{2}\left(q^{2}+M_{\mathrm{PS}}^{\mathrm{unit}^{2}}\right)}{\left(q^{2}+M_{\mathrm{PS}}^{\mathrm{deg}^{2}}\right)^{2}} .
$$

By subtracting and adding $\left(q^{2}+M_{\mathrm{PS}}^{\mathrm{deg}^{2}}\right)^{-1}$ to the first and second terms respectively, this can be rearranged as

$$
\sigma_{\eta^{\prime} \rho}^{\text {tot }} \sim \frac{g_{2}^{2}\left(M_{\mathrm{PS}}^{\mathrm{deg}^{2}}-M_{\mathrm{PS}}^{\mathrm{non}-\mathrm{deg}^{2}}\right)}{\left(q^{2}+M_{\mathrm{PS}}^{\mathrm{non}-\mathrm{deg}^{2}}\right)\left(q^{2}+M_{\mathrm{PS}}^{\mathrm{deg}^{2}}\right)}+\frac{g_{2}^{2}\left(M_{\mathrm{PS}}^{\mathrm{deg}^{2}}-M_{\mathrm{PS}}^{\mathrm{uni}^{2}}\right)}{\left(q^{2}+M_{\mathrm{PS}}^{\mathrm{deg}^{2}}\right)^{2}}
$$

which clearly vanishes when the sea and valence quark masses are equal.

The quenched coupling of a light $\eta^{\prime}$ meson (degenerate with the pion) depicted in figure 4(a) is complemented by an infinite sum of sea-quark loop contributions in pQQCD, the first few of which are depicted in figures 4(b) through (d). Using similar techniques, one can show that the sum of these graphs leads to the propagation of a heavy $\eta^{\prime}$ and upon taking $\mu_{0} \rightarrow \infty$ the contribution of figure 4 vanishes.

We note that the coupling $g_{2}$ introduced in [29] takes the value 0.75 , where in terms of the usual $\omega \rho \pi$ coupling constant, $g_{\omega \rho \pi}=16 \mathrm{GeV}^{-1}, g_{2}^{2}=g_{\omega \rho \pi}^{2} f_{\pi}^{2} / 4$. Introducing $f_{\rho \pi \omega}^{2}=\mu_{\rho} g_{2}^{2}$ (with $\mu_{\rho(\pi)}$ the physical $\rho(\pi)$ mass), the total, non-trivial $\eta^{\prime}$ contribution can be written in the 

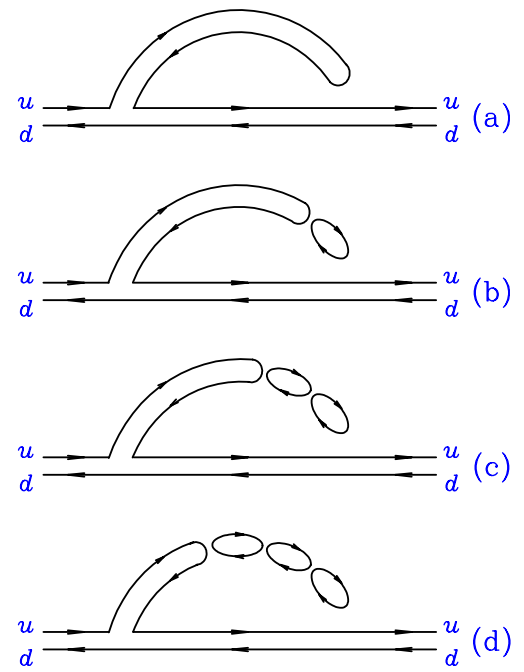

Figure 4. The quenched coupling of a light $\eta^{\prime}$ meson (degenerate with the pion) depicted in (a) is complemented by an infinite sum of sea-quark loop contributions in pQQCD, the first few of which are depicted in (b) through (d).

form (following [27] for large vector meson mass)

$$
\begin{gathered}
\Sigma_{\eta^{\prime} \rho}^{\rho}=\frac{f_{\rho \pi \omega}^{2}}{3 \pi^{2} f_{\pi}^{2}} \int_{0}^{\infty} \frac{k^{4} u^{2}(k) \mathrm{d} k}{\left(k^{2}+\left(M_{\mathrm{PS}}^{\mathrm{non}-\mathrm{deg}}\right)^{2}\right)\left(k^{2}+\left(M_{\mathrm{PS}}^{\mathrm{deg}}\right)^{2}\right)} \times\left(\left(M_{\mathrm{PS}}^{\mathrm{non}-\mathrm{deg}}\right)^{2}-\left(M_{\mathrm{PS}}^{\mathrm{deg}}\right)^{2}\right) \\
+\frac{f_{\rho \pi \omega}^{2}}{3 \pi^{2} f_{\pi}^{2}} \int_{0}^{\infty} \frac{k^{4} u^{2}(k) \mathrm{d} k}{\left(k^{2}+\left(M_{\mathrm{PS}}^{\mathrm{deg}}\right)^{2}\right)^{2}} \times\left(\left(M_{\mathrm{PS}}^{\mathrm{unit}}\right)^{2}-\left(M_{\mathrm{PS}}^{\mathrm{deg}}\right)^{2}\right) .
\end{gathered}
$$

In summary, we find

$$
\Sigma_{\mathrm{TOT}}=\Sigma_{\pi \pi}^{\rho}+\Sigma_{\pi \omega}^{\rho}+\Sigma_{\eta^{\prime} \rho}^{\rho},
$$

where the pion self-energies are given by

$$
\begin{aligned}
& \Sigma_{\pi \pi}^{\rho}=-\frac{f_{\rho \pi \pi}^{2}}{6 \pi^{2}} \int_{0}^{\infty} \frac{k^{4} u_{\pi \pi}^{2}(k) \mathrm{d} k}{\omega_{\pi}(k)\left(\omega_{\pi}^{2}(k)-\mu_{\rho}^{2} / 4\right)}, \\
& \Sigma_{\pi \omega}^{\rho}=-\frac{f_{\rho \pi \omega}^{2}}{3 \pi^{2} f_{\pi}^{2}} \int_{0}^{\infty} \frac{k^{4} u_{\pi \omega}^{2}(k) \mathrm{d} k}{\omega_{\pi}(k)\left(\omega_{\pi}(k)+\Delta M_{\omega \rho}\right)}
\end{aligned}
$$

and

$$
\omega_{\pi}^{2}(k)=k^{2}+\left(M_{\mathrm{PS}}^{\mathrm{non}-\mathrm{deg}}\right)^{2} .
$$

As the $\omega$ meson contributes via a sea-quark loop as illustrated in figure 1, the mass splitting between the $\rho$ and $\omega$ is

$$
\Delta M_{\omega \rho}=M_{V}^{\mathrm{non}-\mathrm{deg}}-M_{V}^{\mathrm{deg}} .
$$

We note that $\left(\omega_{\pi}(k)+\Delta M_{\omega \rho}\right)>0$ for all quark masses and non-trivial momenta considered in the lattice analysis. We use the values $f_{\rho \pi \pi}=6.028$ (obtained from [27]) and $f_{\pi}=92.4 \mathrm{MeV}$. 
We use a standard dipole form factor, which takes the form

$$
\begin{aligned}
& u(k)=\frac{\Lambda^{4}}{\left(\Lambda^{2}+k^{2}\right)^{2}}, \\
& u_{\pi \omega}(k)=u(k), \\
& u_{\pi \pi}(k)=u(k) u^{-1}\left(\sqrt{\mu_{\rho}^{2} / 4-\mu_{\pi}^{2}}\right),
\end{aligned}
$$

where the second factor in $u_{\pi \pi}(k)$ ensures the correct on-shell normalization condition.

To account for finite volume artefacts, the self-energy equations are discretized so that only those momenta allowed on the lattice appear [27, 30]:

$$
4 \pi \int_{0}^{\infty} k^{2} \mathrm{~d} k=\int \mathrm{d}^{3} k \approx \frac{1}{V}\left(\frac{2 \pi}{a}\right)^{3} \sum_{k_{x}, k_{y}, k_{z}},
$$

with

$$
k_{x, y, z}=\frac{2 \pi(i, j, k)}{a N_{x, y, z}} .
$$

The purpose of the finite-range regulator is to regularize the theory as $k_{x}, k_{y}, k_{z}$ tend to infinity. Indeed, once any one of $k_{x}, k_{y}$ or $k_{z}$ is greater than $\sim 10 \Lambda$ the contribution to the integral is negligible, thereby ensuring convergence of the summation. Hence, we would like the highest momentum in each direction to be just over $10 \Lambda$. For practical calculation, we therefore use the following to calculate the maxima and minima for $i, j, k$ :

$$
\begin{aligned}
& (i, j, k)_{\max }=\left[\frac{10 \Lambda a}{2 \pi} N_{(x, y, z)}\right]+1, \\
& (i, j, k)_{\min }=-\left[\frac{10 \Lambda a}{2 \pi} N_{(x, y, z)}\right]-1,
\end{aligned}
$$

where $[. .$.$] denotes the integer part.$

We study a range of values of $\Lambda$, starting with the value, $\Lambda_{\pi \omega}=630 \mathrm{MeV}$, used in [27]. Figure 5 shows the various self-energy contributions, $\Sigma_{\pi \pi}^{\rho}, \Sigma_{\pi \omega}^{\rho}$ and $\Sigma_{\eta^{\prime} \rho}^{\rho}$ as a function of $M_{\mathrm{PS}}^{\text {non-deg }}$ (see equations (7), (8) and (5)) for the representative $\left(\beta, \kappa_{\text {sea }}\right)=(2.10,0.1382)$ dataset. In section 4.3 we perform a highly constrained fit to a large 'global' dataset, and this enables us to determine a best value of $\Lambda$ which minimizes the global $\chi^{2}$.

\section{Overview of CP-PACS data}

In [1], the CP-PACS collaboration published meson spectrum data from dynamical simulations for mean-field improved Wilson fermions with improved gluons at four different $\beta$ values. For each value of $\beta$, ensembles were generated for four different values of $\kappa_{\text {sea }}$, giving a total of 16 independent ensembles. Table 1 summarizes the lattice parameters used. The pseudoscalarto vector-meson mass ratio $M_{\mathrm{PS}}^{\text {unit }} / M_{V}^{\text {unit }}$ (which gives a measure of the mass of the sea quarks used) varies from 0.55 to 0.80 and the lattice spacing, $a$, from around 0.09 to $0.29 \mathrm{fm}$. For each of the 16 ensembles there are five $\kappa_{\mathrm{val}}$ values considered [1]. Thus there are a total of 80 $\left(M_{V}^{\mathrm{deg}}, M_{\mathrm{PS}}^{\mathrm{deg}}\right)$ data points available for analysis. In our study we consider the two cases where the lattice spacing is set using either $r_{0}$ [31] or the string tension $\sigma$.

In the absence of the full set of original CP-PACS data, we generate 1000 bootstrap clusters for all $M_{\mathrm{PS}}$ and $M_{V}$ data using a Gaussian distribution whose central value and full width half maximum (FWHM) are the same as the central values and errors published in table 21 of [1]. Of course, our errors are totally uncorrelated throughout; 


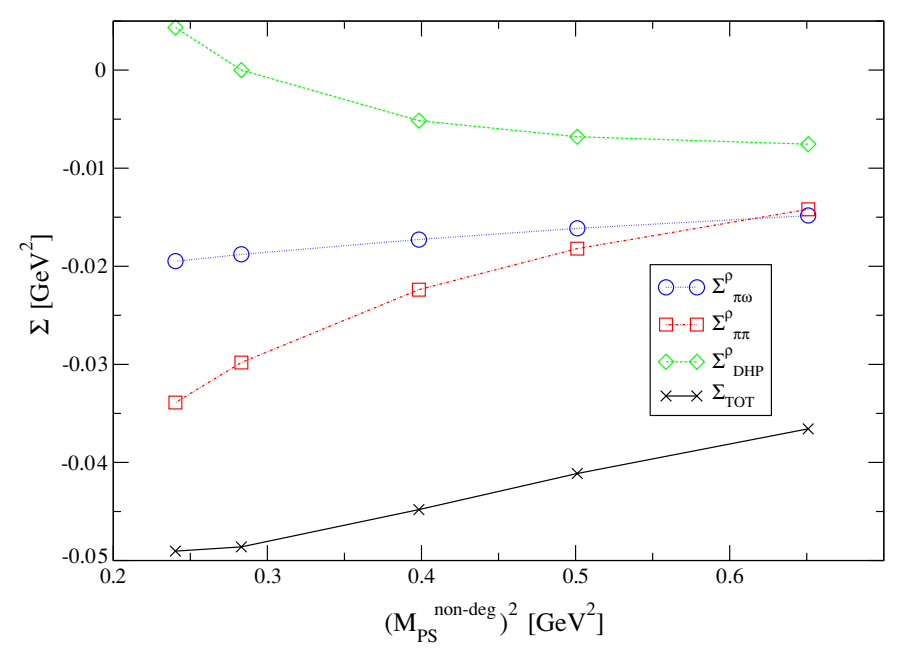

Figure 5. The self-energy contributions (see equations (7), (8) and (5)) versus $M_{\mathrm{PS}}^{\text {non-deg }}$ data for the ensemble $\left(\beta, \kappa_{\text {sea }}\right)=(2.10,0.1382)$ using $\Lambda=630 \mathrm{MeV}$.

Table 1. The lattice parameters of the CP-PACS simulation used in this data analysis, taken from [1]. The superscript 'unit' refers to the unitary data (i.e., where $\kappa_{\text {val }}^{1} \equiv \kappa_{\text {val }}^{2} \equiv \kappa_{\text {sea }}$ ). Note that the errors reported in this table are obtained with our bootstrap ensembles (see text).

\begin{tabular}{llllll}
\hline$\beta$ & $\kappa_{\text {sea }}$ & Volume & $M_{\mathrm{PS}}^{\text {unit }} / M_{V}^{\text {unit }}$ & $a_{r_{0}}(\mathrm{fm})$ & $a_{\sigma}(\mathrm{fm})$ \\
\hline 1.80 & 0.1409 & $12^{3} \times 24$ & $0.8067_{-9}^{+9}$ & $0.286_{-6}^{+6}$ & $0.288_{-3}^{+3}$ \\
1.80 & 0.1430 & $12^{3} \times 24$ & $0.7526_{-15}^{+16}$ & $0.272_{-2}^{+2}$ & $0.280_{-5}^{+4}$ \\
1.80 & 0.1445 & $12^{3} \times 24$ & $0.694_{-2}^{+2}$ & $0.258_{-4}^{+4}$ & $0.269_{-3}^{+2}$ \\
1.80 & 0.1464 & $12^{3} \times 24$ & $0.547_{-4}^{+4}$ & $0.237_{-4}^{+4}$ & $0.248_{-3}^{+2}$ \\
1.95 & 0.1375 & $16^{3} \times 32$ & $0.8045_{-11}^{+11}$ & $0.196_{-4}^{+4}$ & $0.2044_{-12}^{+10}$ \\
1.95 & 0.1390 & $16^{3} \times 32$ & $0.752_{-2}^{+2}$ & $0.185_{-3}^{+3}$ & $0.1934_{-15}^{+14}$ \\
1.95 & 0.1400 & $16^{3} \times 32$ & $0.690_{-2}^{+2}$ & $0.174_{-2}^{+2}$ & $0.1812_{-12}^{+12}$ \\
1.95 & 0.1410 & $16^{3} \times 32$ & $0.582_{-3}^{+3}$ & $0.163_{-2}^{+2}$ & $0.1699_{-15}^{+13}$ \\
2.10 & 0.1357 & $24^{3} \times 48$ & $0.806_{-2}^{+2}$ & $0.1275_{-5}^{+5}$ & $0.1342_{-8}^{+8}$ \\
2.10 & 0.1367 & $24^{3} \times 48$ & $0.755_{-2}^{+2}$ & $0.1203_{-5}^{+4}$ & $0.1254_{-8}^{+8}$ \\
2.10 & 0.1374 & $24^{3} \times 48$ & $0.691_{-3}^{+3}$ & $0.1157_{-4}^{+4}$ & $0.1203_{-6}^{+6}$ \\
2.10 & 0.1382 & $24^{3} \times 48$ & $0.576_{-4}^{+3}$ & $0.1093_{-3}^{+3}$ & $0.1129_{-5}^{+4}$ \\
2.20 & 0.1351 & $24^{3} \times 48$ & $0.799_{-3}^{+3}$ & $0.0997_{-5}^{+4}$ & $0.10503_{-15}^{+15}$ \\
2.20 & 0.1358 & $24^{3} \times 48$ & $0.753_{-4}^{+4}$ & $0.0966_{-4}^{+4}$ & $0.1013_{-2}^{+3}$ \\
2.20 & 0.1363 & $24^{3} \times 48$ & $0.705_{-6}^{+6}$ & $0.0936_{-4}^{+4}$ & $0.0978_{-3}^{+3}$ \\
2.20 & 0.1368 & $24^{3} \times 48$ & $0.632_{-8}^{+8}$ & $0.0906_{-4}^{+4}$ & $0.0949_{-2}^{+2}$ \\
\hline
\end{tabular}

i.e., each $M_{V}\left(\beta, \kappa_{\text {sea }} ; \kappa_{\text {val }}^{1}, \kappa_{\text {val }}^{2}\right)$ bootstrap cluster is uncorrelated with the corresponding $M_{\mathrm{PS}}\left(\beta, \kappa_{\text {sea }} ; \kappa_{\text {val }}^{1}, \kappa_{\text {val }}^{2}\right)$ bootstrap cluster. Furthermore, the $M\left(\beta, \kappa_{\text {sea }} ; \kappa_{\text {val }}^{1}, \kappa_{\text {val }}^{2}\right)$ data are not correlated with the $M\left(\beta^{\prime}, \kappa_{\text {sea }}^{\prime} ; \kappa_{\text {val }}^{1}, \kappa_{\text {val }}^{2}\right)$ data, and nor are the $M\left(\beta, \kappa_{\text {sea }} ; \kappa_{\text {val }}^{1}, \kappa_{\text {val }}^{2}\right)$ data correlated with those for $M\left(\beta, \kappa_{\text {sea }} ; \kappa_{\text {val }}^{1^{\prime}}, \kappa_{\text {val }}^{2^{\prime}}\right)$. 


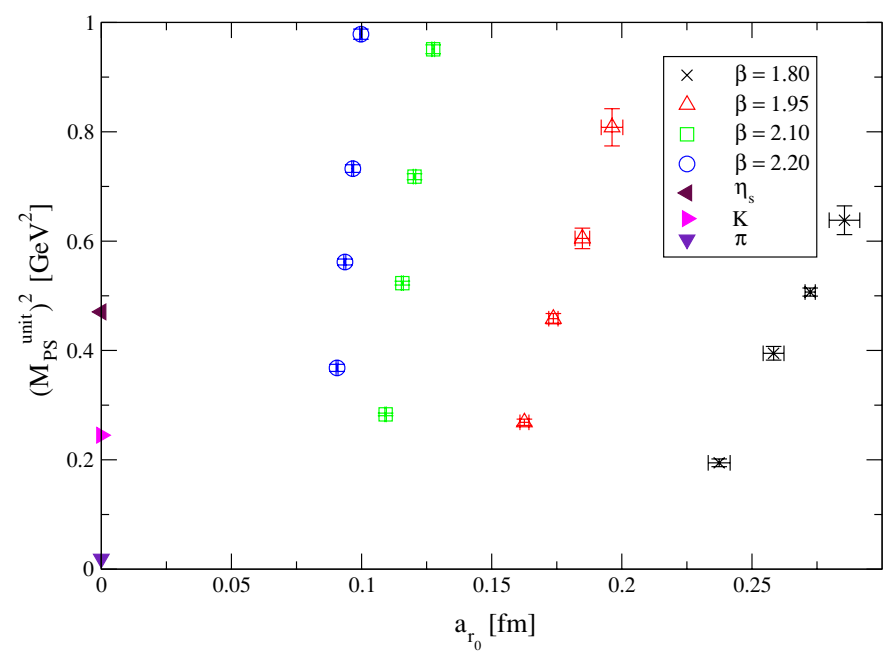

Figure 6. The range of sea-quark mass $\left(M_{\mathrm{PS}}^{\text {unit }}\right)^{2}$ and lattice spacing, $a_{r_{0}}$, covered by the CP-PACS data displayed in table $1 .\left(M_{\mathrm{PS}}^{\text {unit }}\right)^{2}$ is the pseudoscalar meson mass squared at the unitary point, i.e., where $\kappa_{\mathrm{val}} \equiv \kappa_{\text {sea }}$. The experimental points for the $\pi, K$ and ' $\eta_{s}$ ' mesons are also shown for reference.

We expect therefore that the statistical errors of our final results will be overestimates of the true error, since we have not benefited from the partial cancellation of statistical errors which occurs when combining correlated data. We can obtain a rough estimate of the increase in our errors due to the fact that we do not maintain correlations as follows. The ratio $M_{\mathrm{PS}}^{\text {unit }} / M_{V}^{\text {unit }}$ listed in table 1 is obtained from our bootstrap data. Comparing this with the $M_{\mathrm{PS}}^{\text {unit }} / M_{V}^{\text {unit }}$ data in table 21 of [1] (which benefits from the cancellation of correlations), we see that a very rough estimate of the effect of ignoring correlations is to increase the errors by $\sim 20 \%$. We expect that a similar increase in errors will apply to other quantities.

The lattice spacings $a_{r_{0}, \sigma}$ were obtained from table 12 of [1] using $r_{0}=0.49 \mathrm{fm}$ and $\sqrt{\sigma}=440 \mathrm{MeV}$. Again we generated 1000 bootstrap clusters with a Gaussian distribution, as in the meson mass data. Figure 6 shows the unitary (i.e., $\kappa_{\text {val }}^{1} \equiv \kappa_{\text {val }}^{2} \equiv \kappa_{\text {sea }}$ ) pseudoscalar mass plotted against the lattice spacing, $a_{r_{0}}$, for the 16 ensembles in table 1 . (Note that $\left(M_{\mathrm{PS}}^{\text {unit }}\right)^{2}$ is a direct measure of the sea-quark mass since, from PCAC, $\left.\left(M_{\mathrm{PS}}^{\text {unit }}\right)^{2} \propto m_{\text {sea. }}^{q}\right)$ Also shown for reference are the physical pseudoscalar mesons $\pi, K$ and ' $\eta_{s}$ '. Note the large range of both the lattice spacing $a_{r_{0}}$ and $m_{\text {sea }}^{q}$ in the simulations, and that the lattice spacing is primarily determined by the $\beta$ value, rather than the value of $m_{\text {sea }}^{q}$.

Because $r_{0}$ or $\sigma$ are only mildly dependent on the quark mass, it is convenient to define one of them as a renormalization scale, independent of quark mass, such that the effective theory of QCD at unphysical quark masses can be related to that at the physical point. Choosing such a definition removes any ambiguity associated with attempting to set the scale from the result of an extrapolation to a point which has not been directly simulated. The observed variation of $\left(M_{\mathrm{PS}}^{\text {unit }}\right)^{2}$ with $\beta$, or $a_{r_{0}}$ with $\kappa$, in figure 6 should not be confused with scaling violations, but simply highlights the intimate relation between $\beta$ and $\kappa$ in dynamical simulations.

The physical volume for these 16 ensembles is $L a \approx 2.5 \mathrm{fm}$ for the $\beta=1.80,1.95$ and 2.10 cases, but the $\beta=2.20$ ensemble had a slightly smaller physical volume. The associated finite volume effects are incorporated through evaluating the chiral loops by explicitly summing the discrete pion momenta allowed on the lattice. We treat all 16 ensembles on an equal footing. 
The finite-volume effects are corrected when making contact with the physical observables by evaluating the chiral loop integrals with continuous loop momenta.

The action used in [1] is mean-field improved, rather than non-perturbatively improved, and will therefore have some residual lattice systematic errors of $\mathcal{O}(a)$ [32]. We fit the data assuming both $\mathcal{O}(a)$ and $\mathcal{O}\left(a^{2}\right)$ effects in sections 4.2 and 4.3 , and are thus able to obtain continuum predictions. Our empirical analysis suggests that non-analytic terms generated in a dual expansion of both $a$ and $m_{q}$ [33-38] are either small or can be absorbed at present into the $\mathcal{O}(a)$ and $\mathcal{O}\left(a^{2}\right)$ effects considered here.

\section{Fitting analysis}

\subsection{Summary of analysis techniques}

Our chiral extrapolation approach is based upon converting all masses into physical units prior to any extrapolation being performed. An alternative approach would be to extrapolate dimensionless masses (in lattice units) [1]. However, using physical units offers the following key advantages:

- The data from different ensembles can be combined in a global fit. If the masses were left in dimensionless units, there would be no possibility of combining data at different lattice spacings together into such a global fit.

- Dimensionful mass predictions from lattice simulations are effectively mass ratios, and hence one would expect some of the systematic (and statistical) errors to cancel. That is, $M^{\text {dimful }}=M^{\#} \times a^{-1} \equiv M^{\#} / M_{\Omega}^{\#} \times M_{\Omega}^{\text {expt }}$, where $\Omega$ is the quantity used to set the lattice spacing, $a$, the superscripts '\#', 'expt' refer to the lattice mass estimate and the experimental value, respectively.

- Lattice data are not in the regime where the coefficients of non-analytic terms in the chiral expansion can be reliably constrained. These dimensionful quantities must therefore be fixed to their phenomenological values.

In this paper we use two different quantities for setting the scale, $r_{0}$ and $\sigma$, although we have a preference, as explained in section 4.3, for $r_{0}$. Table 1 lists values for $a_{r_{0}}$ and $a_{\sigma}$. In converting results into physical units, we use the lattice spacing as directly measured for each gauge field ensemble.

In our chiral extrapolations we use two basic fitting functions, the finite-range regularization method (hereafter referred to as the 'Adelaide' method)

$$
\sqrt{\left(M_{V}^{\mathrm{deg}}\right)^{2}-\Sigma_{\mathrm{TOT}}}=a_{0}+a_{2}\left(M_{\mathrm{PS}}^{\mathrm{deg}}\right)^{2}+a_{4}\left(M_{\mathrm{PS}}^{\mathrm{deg}}\right)^{4}+a_{6}\left(M_{\mathrm{PS}}^{\mathrm{deg}}\right)^{6},
$$

where $\Sigma_{\text {TOT }}$ is calculated using equation (6), and a naive polynomial fit

$$
M_{V}^{\mathrm{deg}}=a_{0}+a_{2}\left(M_{\mathrm{PS}}^{\mathrm{deg}}\right)^{2}+a_{4}\left(M_{\mathrm{PS}}^{\mathrm{deg}}\right)^{4}+a_{6}\left(M_{\mathrm{PS}}^{\mathrm{deg}}\right)^{6} .
$$

In each case we refer to these fits as 'cubic' since they include cubic terms in the chiral expansion of $m_{q} \propto\left(M_{\mathrm{PS}}\right)^{2}$. We also perform fits with the coefficient $a_{6}$ set to zero in equations (13) and (14), referring to these as 'quadratic'. It is worth noting that the dominant functional form of $M_{V}$ with $\left(M_{\mathrm{PS}}^{\mathrm{deg}}\right)^{2}$ is linear (see, for example, figure 7, where the LHS of equations (13) and (14) are referred to as $\left.M_{V}^{\text {sub }}\right)$. We exploit this fact in the fitting functions given above. In particular, this is why the Adelaide fit uses $\sqrt{\left(M_{V}^{\mathrm{deg}}\right)^{2}-\Sigma_{\text {TOT }}}$ on the LHS rather than $\left(M_{V}^{\mathrm{deg}}\right)^{2}-\Sigma_{\mathrm{TOT}}$ which would, a priori, be an equally valid chiral expansion. Thus, with the above functional forms, we expect the coefficients, $a_{n}$, to be small for $n \geqslant 4$ and this is, in fact, what we find. 


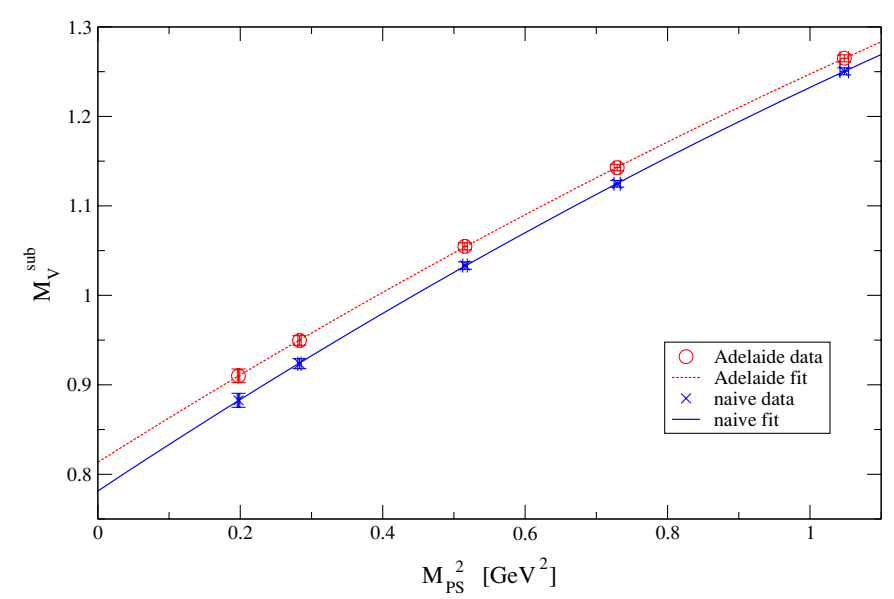

Figure 7. A plot of $M_{V}^{\text {Sub }}$ versus $M_{\mathrm{PS}}$ data for the ensemble $\left(\beta, \kappa_{\text {sea }}\right)=(2.10,0.1382)$ together with the results of the quadratic Adelaide, equation (13), and naive, equation (14), fits. $M_{V}^{\text {Sub }}$ is defined as $M_{V}^{\text {Sub }}=\sqrt{\left(M_{V}^{\text {deg }}\right)^{2}-\Sigma_{\mathrm{TOT}}}$ for the Adelaide fit (i.e., the LHS of equation 13-note that $\Sigma_{\text {TOT }}$ is negative), and $M_{V}^{\text {Sub }}=M_{V}^{\text {deg }}$ for the naive fit (i.e., the LHS of equation (14)).

In the following, we first use equations (13) and (14) to fit the 16 ensembles in table 1 separately. We then turn to a global fit, where all 16 ensembles are combined in one fitting function.

\subsection{Individual ensemble fits}

We begin our analysis by fitting the meson spectrum of each of the 16 ensembles listed in table 1 separately. In this section we use $r_{0}$ to set the scale and select a value of $\Lambda=650 \mathrm{MeV}$ (see section 4.3). We use both the Adelaide, equation (13), and naive, equation (14), fitting functions and restrict our attention to quadratic $\left(a_{6} \equiv 0\right)$ chiral fits, since there are only five $\left(M_{V}^{\mathrm{deg}}, M_{\mathrm{PS}}^{\mathrm{deg}}\right)$ data points available for each ensemble. The coefficients, $a_{0,2,4}$, obtained by fitting $M_{V}$ against $M_{\mathrm{PS}}$ with both the naive (equation (14)) and Adelaide (equation (13)) fitting functions are listed in table 2 . We see that the $a_{4}$ coefficients are both small and generally poorly determined, confirming our decision to fit to the quadratic, rather than the cubic, chiral extrapolation form. We note also that there is some agreement between the naive and Adelaide $a_{0,2}$ coefficients, although their variation with $\kappa_{\text {sea }}$ tends to be different.

Figure 7 shows the results of these fits for the $\left(\beta, \kappa_{\text {sea }}\right)=(2.10,0.1382)$ ensemble, which is a good representative of all of them. The scale is set from $r_{0}$, which is our preferred method (see section 4.3). Note that this ensemble's $\left(a, m_{\text {sea }}^{q}\right)$ coordinates are closest to the physical point $\left(a, m_{\text {sea }}^{q}\right)=\left(0, m_{u, d}\right)$ for those ensembles with $L a \approx 2.5 \mathrm{fm}$ (see figure 6$)$.

The values of $a_{0,2}$ in table 2 hint at a systematic variation of $a_{0,2}$ with $a_{r_{0}}$. To check this we plot $a_{0}$ and $a_{2}$ against $a_{r_{0}}$ (for both the linear and Adelaide fits) in figures 8 and 9. This motivates a continuum extrapolation of the form

$$
a_{0,2}=a_{0,2}^{\text {cont }}+X_{0,2}^{\text {individual }} a_{r_{0}} .
$$

The results of these fits are displayed in table 3. From the values of $X_{0,2}^{\text {individual }}$ in table 3 , we can confirm statistically significant $\mathcal{O}(a)$ effects in $a_{0}$ but not in $a_{2}$. This will be important in determining our fit procedure in section 4.3. 


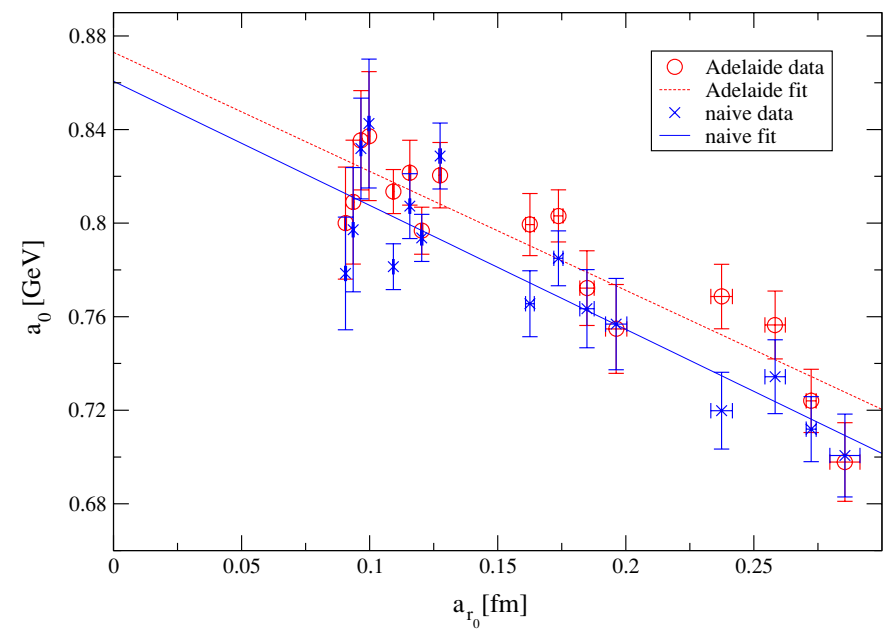

Figure 8. A continuum extrapolation of the $a_{0}$ coefficient obtained from both the Adelaide and naive fits using equation (15).

Table 2. The coefficients obtained from fitting $M_{V}$ data against $M_{\mathrm{PS}}^{2}$ using both the naive and Adelaide fits (i.e., equations (14) and (13), respectively) for each of the 16 ensembles listed in table 1. As discussed in the text, we restrict these fits to quadratic rather than cubic chiral functions (i.e., $a_{6} \equiv 0$ ). The scale was set using $r_{0}$.

\begin{tabular}{llllllll}
\hline$\beta$ & $\kappa_{\text {sea }}$ & $a_{0}^{\text {naive }}(\mathrm{GeV})$ & $a_{0}^{\text {Adel }}(\mathrm{GeV})$ & $a_{2}^{\text {naive }}\left(\mathrm{GeV}^{-1}\right)$ & $a_{2}^{\text {Adel }}\left(\mathrm{GeV}^{-1}\right)$ & $a_{4}^{\text {naive }}\left(\mathrm{GeV}^{-3}\right)$ & $a_{4}^{\text {Adel }}\left(\mathrm{GeV}^{-3}\right)$ \\
\hline 1.80 & 0.1409 & $0.701_{-22}^{+14}$ & $0.70_{-2}^{+2}$ & $0.46_{-3}^{+7}$ & $0.54_{-5}^{+5}$ & $-0.01_{-7}^{+3}$ & $-0.09_{-5}^{+5}$ \\
1.80 & 0.1430 & $0.712_{-13}^{+14}$ & $0.724_{-13}^{+14}$ & $0.48_{-6}^{+6}$ & $0.51_{-6}^{+5}$ & $-0.04_{-6}^{+6}$ & $-0.08_{-6}^{+6}$ \\
1.80 & 0.1445 & $0.73_{-2}^{+2}$ & $0.756_{-15}^{+14}$ & $0.43_{-5}^{+5}$ & $0.44_{-5}^{+5}$ & $0.01_{-5}^{+5}$ & $-0.01_{-5}^{+5}$ \\
1.80 & 0.1464 & $0.72_{-2}^{+2}$ & $0.769_{-15}^{+13}$ & $0.49_{-5}^{+5}$ & $0.43_{-5}^{+5}$ & $-0.02_{-6}^{+6}$ & $0.007_{-58}^{+59}$ \\
1.95 & 0.1375 & $0.76_{-2}^{+2}$ & $0.75_{-2}^{+2}$ & $0.49_{-4}^{+4}$ & $0.53_{-4}^{+4}$ & $-0.05_{-3}^{+4}$ & $-0.08_{-3}^{+3}$ \\
1.95 & 0.1390 & $0.76_{-2}^{+2}$ & $0.772_{-15}^{+17}$ & $0.47_{-4}^{+4}$ & $0.49_{-4}^{+4}$ & $-0.03_{-3}^{+4}$ & $-0.05_{-4}^{+4}$ \\
1.95 & 0.1400 & $0.785_{-12}^{+12}$ & $0.803_{-11}^{+11}$ & $0.43_{-4}^{+4}$ & $0.44_{-4}^{+4}$ & $-0.01_{-3}^{+3}$ & $-0.02_{-3}^{+3}$ \\
1.95 & 0.1410 & $0.766_{-15}^{+13}$ & $0.799_{-14}^{+13}$ & $0.48_{-4}^{+5}$ & $0.45_{-4}^{+5}$ & $-0.03_{-4}^{+4}$ & $-0.03_{-4}^{+3}$ \\
2.10 & 0.1357 & $0.829_{-14}^{+14}$ & $0.820_{-14}^{+14}$ & $0.42_{-4}^{+5}$ & $0.46_{-4}^{+5}$ & $-0.02_{-4}^{+3}$ & $-0.05_{-4}^{+3}$ \\
2.10 & 0.1367 & $0.794_{-10}^{+11}$ & $0.797_{-10}^{+11}$ & $0.50_{-3}^{+3}$ & $0.53_{-3}^{+3}$ & $-0.06_{-3}^{+3}$ & $-0.08_{-2}^{+3}$ \\
2.10 & 0.1374 & $0.807_{-14}^{+13}$ & $0.822_{-14}^{+13}$ & $0.48_{-4}^{+4}$ & $0.49_{-4}^{+4}$ & $-0.05_{-3}^{+3}$ & $-0.06_{-3}^{+3}$ \\
2.10 & 0.1382 & $0.781_{-9}^{+10}$ & $0.814_{-9}^{+10}$ & $0.53_{-3}^{+3}$ & $0.50_{-3}^{+3}$ & $-0.08_{-2}^{+2}$ & $-0.07_{-2}^{+2}$ \\
2.20 & 0.1351 & $0.84_{-3}^{+3}$ & $0.84_{-3}^{+3}$ & $0.43_{-8}^{+8}$ & $0.46_{-8}^{+8}$ & $-0.02_{-6}^{+6}$ & $-0.04_{-6}^{+6}$ \\
2.20 & 0.1358 & $0.83_{-2}^{+2}$ & $0.84_{-2}^{+2}$ & $0.44_{-7}^{+7}$ & $0.46_{-7}^{+7}$ & $-0.03_{-5}^{+5}$ & $-0.05_{-5}^{+5}$ \\
2.20 & 0.1363 & $0.80_{-3}^{+3}$ & $0.81_{-3}^{+3}$ & $0.51_{-8}^{+8}$ & $0.52_{-8}^{+8}$ & $-0.07_{-6}^{+6}$ & $-0.08_{-6}^{+6}$ \\
2.20 & 0.1368 & $0.78_{-2}^{+2}$ & $0.80_{-2}^{+2}$ & $0.52_{-8}^{+8}$ & $0.51_{-8}^{+7}$ & $-0.06_{-6}^{+6}$ & $-0.06_{-6}^{+6}$ \\
\hline & & & & & &
\end{tabular}

\subsection{Global fits}

We now turn our attention to an analysis of the whole (degenerate) data set. Figure 10 shows all of the degenerate CP-PACS data with the physical scale set using $r_{0}$. Since there are 16 


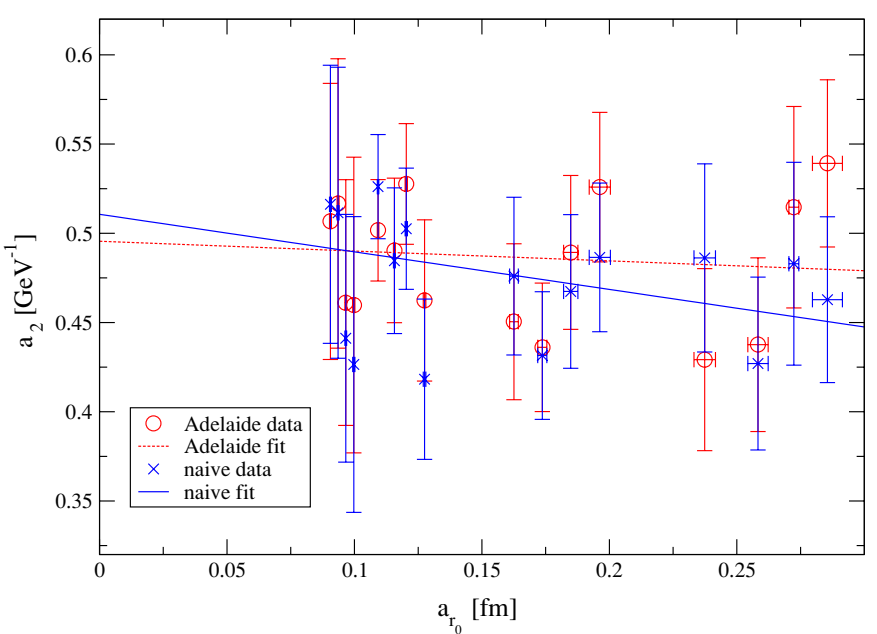

Figure 9. A continuum extrapolation of the $a_{2}$ coefficient obtained from both the Adelaide and naive fits using equation (15).

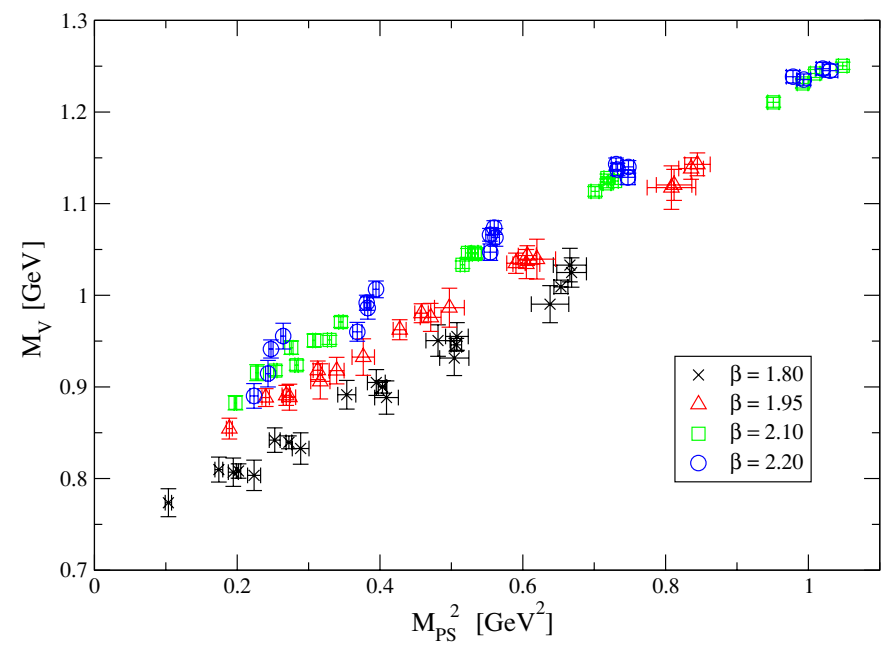

Figure 10. Illustration of the 80 lattice data points of the degenerate CP-PACS data set, with the physical scale of $\mathrm{a}_{r_{0}}$.

Table 3. The coefficients obtained from the continuum extrapolation of both the naive and Adelaide fits, using the values of $a_{0,2}$ given in table 2 with equation (15).

\begin{tabular}{lllllll}
\hline & $a_{0}^{\text {cont. }}(\mathrm{GeV})$ & $X_{0}^{\text {individual }}\left(\mathrm{GeV} \mathrm{fm}^{-1}\right)$ & $\chi_{0}^{2} /$ d.o.f. & $a_{2}^{\text {cont. }}\left(\mathrm{GeV}^{-1}\right)$ & $X_{2}^{\text {individual }}\left(\mathrm{GeV}^{-1} \mathrm{fm}^{-1}\right)$ & $\chi_{2}^{2} /$ d.o.f. \\
\hline Naive fit & $0.861_{-9}^{+11}$ & $-0.53_{-7}^{+5}$ & $21 / 14$ & $0.51_{-4}^{+3}$ & $-0.21_{-15}^{+23}$ & $8 / 14$ \\
Adelaide fit & $0.873_{-10}^{+10}$ & $-0.51_{-6}^{+5}$ & $16 / 14$ & $0.50_{-3}^{+3}$ & $-0.06_{-18}^{+19}$ & $10 / 14$ \\
\hline
\end{tabular}

ensembles with five $\left(M_{V}^{\mathrm{deg}}, M_{\mathrm{PS}}^{\mathrm{deg}}\right)$ values in each (see section 3), this global fit contains 80 data points. We expect that this large number of data points should produce a highly constrained set of fit parameters, $a_{0,2, \ldots}$. 
Table 4. The different fit types used in the global analysis. Fits for each of the $2^{4}$ choices depicted above were performed.

\begin{tabular}{|c|c|c|c|}
\hline Approach & $\begin{array}{l}\text { Chiral } \\
\text { extrapolation }\end{array}$ & $\begin{array}{l}\text { Treatment of lattice } \\
\text { spacing artefacts in } a_{0}\end{array}$ & $\begin{array}{l}\text { Lattice spacing } \\
\text { set from }\end{array}$ \\
\hline $\begin{array}{l}\text { Adelaide } \\
\text { (i.e., equation (16)) }\end{array}$ & $\begin{array}{l}\text { Cubic } \\
\text { (i.e. } \mathcal{O}\left(M_{\mathrm{PS}}^{6}\right) \text { included) }\end{array}$ & $\begin{array}{l}a_{0} \text { term has } \\
\mathcal{O}\left(a+a^{2}\right) \text { corrections }\end{array}$ & $r_{0}$ \\
\hline $\begin{array}{l}\text { Naive } \\
\text { (i.e., equation (17)) }\end{array}$ & $\begin{array}{l}\text { Quadratic } \\
\text { (i.e. no } \mathcal{O}\left(M_{\mathrm{PS}}^{6}\right) \text { term) }\end{array}$ & $\begin{array}{l}a_{0} \text { term has } \\
\text { only } \mathcal{O}\left(a^{2}\right) \text { corrections }\end{array}$ & $\sigma$ \\
\hline
\end{tabular}

Referring to the coefficients listed in table 2 and the discussion in the previous section, we observe a significant variation amongst the $a_{0}$ values with lattice spacing, whereas the values of $a_{2}$ are approximately constant with lattice spacing. We also recall that the $a_{4}$ coefficient was undetermined. This suggests that we should allow for some variation of the $a_{0}$ coefficient with lattice spacing and in consequence we adopt the following modified version of the Adelaide and naive fitting functions, based on equations (13) and (14),

$\sqrt{\left(M_{V}^{\mathrm{deg}}\right)^{2}-\Sigma_{\mathrm{TOT}}}=\left(a_{0}^{\mathrm{cont}}+X_{1} a+X_{2} a^{2}\right)+a_{2}\left(M_{\mathrm{PS}}^{\mathrm{deg}}\right)^{2}+a_{4}\left(M_{\mathrm{PS}}^{\mathrm{deg}}\right)^{4}+a_{6}\left(M_{\mathrm{PS}}^{\mathrm{deg}}\right)^{6}$

and

$M_{V}^{\mathrm{deg}}=\left(a_{0}^{\mathrm{cont}}+X_{1} a+X_{2} a^{2}\right)+a_{2}\left(M_{\mathrm{PS}}^{\mathrm{deg}}\right)^{2}+a_{4}\left(M_{\mathrm{PS}}^{\mathrm{deg}}\right)^{4}+a_{6}\left(M_{\mathrm{PS}}^{\mathrm{deg}}\right)^{6}$,

respectively. As in the previous section we refer to the above fits as 'cubic', since they include the $a_{6}$ term (which is proportional to $m_{q}^{3}$ ). As above we also perform fits with $a_{6}$ set to zero, referring to these as 'quadratic'. These fitting functions, equations (16) and (17), have both $\mathcal{O}(a)$ and $\mathcal{O}\left(a^{2}\right)$ terms, because the lattice action used is only mean-field improved and will contain $\mathcal{O}\left(a^{2}\right)$ errors, together with some residual errors at $\mathcal{O}(a)$. In the following we will experiment by turning off the $\mathcal{O}(a)$ term (i.e. by setting $X_{1} \equiv 0$ ) in equations (16) and (17) in order to see whether these residual $\mathcal{O}(a)$ errors are significant. Note that we also included $\mathcal{O}\left(a, a^{2}\right)$ terms in $a_{2}$ (and even $a_{4}$ ) as a check but found that these fits were unstable, confirming the findings of the previous section that there are discernible lattice spacing effects only in the $a_{0}$ coefficient.

The global fits used both $r_{0}$ and the string tension, $\sigma$, to set the scale. Thus we have a large number of fit types which are summarized in table 4 . Indeed, there are two choices from each of the four columns in table 4 , making a total of $2^{4}$ fitting procedures. In the following all $2^{4}$ fits were performed. As noted above, the global fits contain 80 data points, and the largest number of fitting parameters is six ( $a_{0}^{\text {cont }}, X_{1}, X_{2}, a_{2}, a_{4}$ and $\left.a_{6}\right) .^{5}$ Thus the global fits are highly constrained.

Before presenting results from the global fits, we recall our discussion of the $\Lambda$ parameter in section 2. The Adelaide approach motivates the introduction of the mass scale, $\Lambda$, as corresponding to the physical size of the pion source in the hadron which controls the chiral physics. It serves to separate the region where chiral physics is important from that where the internal structure of the hadron, which is not part of the effective field theory, becomes dominant. Since we are performing a highly constrained fit procedure, we are able to derive the best $\Lambda$ value from the data as follows. Figure 11 shows the $\chi^{2} /$ d.o.f. as a function of $\Lambda$ and we see that for the results where $r_{0}$ was used to set the scale, all four fitting types display near identical $\chi^{2}$ behaviour, with a distinct minimum at $\Lambda \approx 650 \mathrm{MeV}$. In other words, the $\chi^{2}$ behaviour is independent of whether we chirally expand to $\mathcal{O}\left(M_{\mathrm{PS}}^{4}\right)$ or $\mathcal{O}\left(M_{\mathrm{PS}}^{6}\right)$ or whether we allow for lattice systematics in the $a_{0}$ coefficient of $\mathcal{O}\left(a+a^{2}\right)$ or $\mathcal{O}\left(a^{2}\right)$.

5 The Adelaide approach also involves the $\Lambda$ parameter which is discussed shortly. 


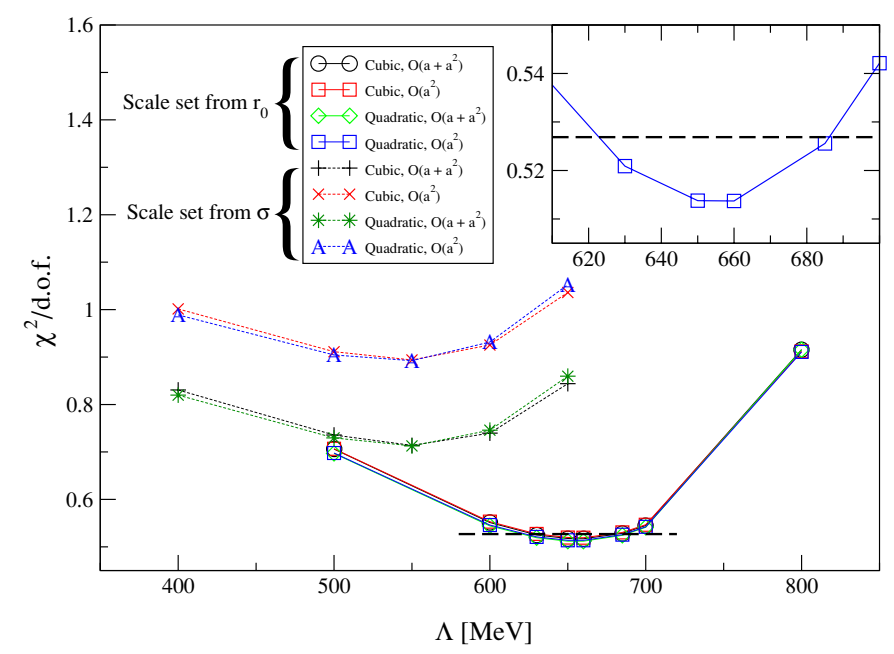

Figure 11. Variation of the $\chi^{2} /$ d.o.f against $\Lambda$. The inset shows a close-up of the minimum for the quadratic fit with $\mathcal{O}\left(a^{2}\right)$ errors using $r_{0}$ to set the scale (i.e. the preferred fitting procedure). The dashed horizontal line represents an increase of $\chi^{2}$ from its minimum value by unity for this fit procedure (i.e. it represents one standard deviation) (see section 5). The intercept of this dashed line with the $\chi^{2}$ curves (at $\Lambda=620$ and $690 \mathrm{MeV}$ ) is used to derive the range of acceptable $\Lambda$ values.

For the case where $\sigma$ is used to set the scale, figure 11 shows that there is a distinct minimum at $\Lambda \approx 550 \mathrm{MeV}$ for all four fitting types. All other things being equal, there is no preference between the cubic or quadratic chiral fits. However, the plot clearly shows that the $\mathcal{O}\left(a+a^{2}\right)$ fits are favoured in that they produce a lower $\chi^{2}$ value than the pure $\mathcal{O}\left(a^{2}\right)$ fits. As a test, we have also fitted the data with a pure $\mathcal{O}(a)$ correction (i.e., equation (16) with $X_{2}=0$ ) and found that the $\chi^{2}$ values for this fit overlay those from the $\mathcal{O}\left(a+a^{2}\right)$ fits. This is strongly suggestive that the dominant lattice-spacing systematic is $\mathcal{O}(a)$ when the string tension is used to set the scale.

We now use these values of $\Lambda$ (550 MeV and $650 \mathrm{MeV}$ for the $\sigma$ and $r_{0}$ cases, respectively) to perform the 16 global fits discussed above and listed in table 4 . Table 5 gives the coefficients and the $\chi^{2} /$ d.o.f. for each fit. Summarizing the results of table 5 and figure 11 (and referring to the $2^{4}$ different fit types listed in table 4 ) we note the following:

Fit approach. The Adelaide method always gives a smaller $\chi^{2}$ than the naive approach, confirming it as the preferred chiral extrapolation procedure.

Chiral extrapolation. The cubic chiral extrapolation (i.e., introducing the $\mathcal{O}\left(M_{\mathrm{PS}}^{6}\right)$ term in equations (16) and (17)) leads to a poorly determined $a_{6}$ coefficient in all cases. Furthermore, it causes the $a_{4}$ coefficient to become much more poorly determined than it is in the quadratic chiral extrapolation cases.

Treatment of lattice spacing artefacts in $a_{0}$. From table 5 , when the scale is set from $r_{0}$, the $a_{0}$ and $a_{2}$ coefficients (which are the dominant terms in the chiral extrapolation) do not depend on whether $\mathcal{O}\left(a+a^{2}\right)$ or $\mathcal{O}\left(a^{2}\right)$ - only corrections are applied to the $a_{0}$ coefficient. Indeed, when $\mathcal{O}\left(a^{2}\right)$ only corrections are used, the error in $a_{0}$ is reduced. However, when the scale is set from $\sigma, a_{0}$ does depend on how the $\mathcal{O}(a)$ systematics are treated. Note also that $X_{1}$ (i.e. the $\mathcal{O}(a)$ coefficient) in the $\sigma$ fits are 2-3 times larger than those from the $r_{0}$ fits. This supports 
Table 5. The results of the global fit analysis. Fits for all $2^{4}$ combinations depicted in table 4 are shown.

\begin{tabular}{|c|c|c|c|c|c|c|c|c|}
\hline $\begin{array}{l}\text { Fit } \\
\text { approach }\end{array}$ & $\begin{array}{l}\text { Scale } \\
\text { from }\end{array}$ & $\begin{array}{l}a_{0}^{\text {cont }} \\
(\mathrm{GeV})\end{array}$ & $\begin{array}{l}X_{1} \\
\left(\mathrm{GeVfm}^{-1}\right)\end{array}$ & $\begin{array}{l}X_{2} \\
\left(\mathrm{GeVfm}^{-2}\right)\end{array}$ & $\begin{array}{l}a_{2} \\
\left(\mathrm{GeV}^{-1}\right)\end{array}$ & $\begin{array}{l}a_{4} \\
\left(\mathrm{GeV}^{-3}\right)\end{array}$ & $\begin{array}{l}a_{6} \\
\left(\mathrm{GeV}^{-5}\right)\end{array}$ & $\begin{array}{l}\chi^{2} / \\
\text { d.o.f. }\end{array}$ \\
\hline & \multicolumn{8}{|c|}{ Cubic chiral extrapolation; $a_{0}$ contains $\mathcal{O}\left(a+a^{2}\right)$} \\
\hline Adelaide & $r_{0}$ & $0.844_{-16}^{+13}$ & $-0.11_{-13}^{+15}$ & $-1.1_{-4}^{+3}$ & $0.47_{-4}^{+5}$ & $-0.02_{-10}^{+8}$ & $-0.02_{-4}^{+5}$ & $38 / 74$ \\
\hline Adelaide & $\sigma$ & $0.836_{-11}^{+9}$ & $-0.37_{-9}^{+10}$ & $-0.2_{-3}^{+2}$ & $0.44_{-4}^{+5}$ & $0.04_{-9}^{+7}$ & $-0.06_{-4}^{+5}$ & $53 / 74$ \\
\hline Naive & $r_{0}$ & $0.819_{-17}^{+13}$ & $-0.15_{-13}^{+15}$ & $-1.1_{-4}^{+3}$ & $0.56_{-5}^{+6}$ & $-0.16_{-10}^{+8}$ & $0.05_{-4}^{+5}$ & $77 / 74$ \\
\hline \multirow[t]{2}{*}{ Naive } & $\sigma$ & $0.805_{-12}^{+10}$ & $-0.38_{-9}^{+11}$ & $-0.3_{-3}^{+2}$ & $0.57_{-4}^{+5}$ & $-0.18_{-10}^{+8}$ & $0.06_{-5}^{+6}$ & $73 / 74$ \\
\hline & \multicolumn{8}{|c|}{ Cubic chiral extrapolation; $a_{0}$ contains $\mathcal{O}\left(a^{2}\right)$ only } \\
\hline Adelaide & $r_{0}$ & $0.835_{-9}^{+8}$ & - & $-1.40_{-4}^{+3}$ & $0.48_{-4}^{+5}$ & $-0.03_{-10}^{+8}$ & $-0.02_{-4}^{+5}$ & $39 / 75$ \\
\hline Adelaide & $\sigma$ & $0.807_{-8}^{+6}$ & - & $-1.24_{-3}^{+3}$ & $0.43_{-4}^{+5}$ & $0.06_{-9}^{+8}$ & $-0.06_{-4}^{+5}$ & $67 / 75$ \\
\hline Naive & $r_{0}$ & $0.806_{-10}^{+8}$ & - & $-1.49_{-4}^{+4}$ & $0.56_{-5}^{+6}$ & $-0.17_{-10}^{+8}$ & $0.06_{-4}^{+5}$ & $78 / 75$ \\
\hline \multirow[t]{2}{*}{ Naive } & $\sigma$ & $0.775_{-8}^{+7}$ & - & $-1.31_{-4}^{+4}$ & $0.56_{-4}^{+5}$ & $-0.16_{-10}^{+8}$ & $0.05_{-5}^{+5}$ & $87 / 75$ \\
\hline & \multicolumn{8}{|c|}{ Quadratic chiral extrapolation; $a_{0}$ contains $\mathcal{O}\left(a+a^{2}\right)$} \\
\hline Adelaide & $r_{0}$ & $0.840_{-12}^{+10}$ & $-0.11_{-13}^{+14}$ & $-1.1_{-4}^{+3}$ & $0.493_{-11}^{+12}$ & $-0.061_{-9}^{+8}$ & - & $38 / 75$ \\
\hline Adelaide & $\sigma$ & $0.829_{-9}^{+8}$ & $-0.37_{-9}^{+10}$ & $-0.2_{-3}^{+2}$ & $0.490_{-11}^{+13}$ & $-0.052_{-11}^{+10}$ & - & $54 / 75$ \\
\hline Naive & $r_{0}$ & $0.828_{-13}^{+11}$ & $-0.16_{-13}^{+15}$ & $-1.1_{-4}^{+3}$ & $0.505_{-11}^{+13}$ & $-0.068_{-10}^{+9}$ & - & $78 / 75$ \\
\hline \multirow[t]{2}{*}{ Naive } & $\sigma$ & $0.812_{-9}^{+8}$ & $-0.37_{-9}^{+11}$ & $-0.3_{-3}^{+2}$ & $0.523_{-12}^{+13}$ & $-0.075_{-11}^{+11}$ & - & $74 / 75$ \\
\hline & \multicolumn{8}{|c|}{ Quadratic chiral extrapolation; $a_{0}$ contains $\mathcal{O}\left(a^{2}\right)$ only } \\
\hline Adelaide & $r_{0}$ & $0.832_{-4}^{+4}$ & - & $-1.40_{-4}^{+3}$ & $0.494_{-11}^{+12}$ & $-0.061_{-9}^{+8}$ & - & $39 / 76$ \\
\hline Adelaide & $\sigma$ & $0.799_{-4}^{+3}$ & - & $-1.23_{-3}^{+3}$ & $0.486_{-11}^{+13}$ & $-0.046_{-11}^{+10}$ & - & $68 / 76$ \\
\hline Naive & $r_{0}$ & $0.815_{-4}^{+4}$ & - & $-1.49_{-4}^{+4}$ & $0.506_{-11}^{+12}$ & $-0.068_{-10}^{+8}$ & - & $79 / 76$ \\
\hline Naive & $\sigma$ & $0.781_{-4}^{+3}$ & - & $-1.31_{-4}^{+3}$ & $0.520_{-12}^{+13}$ & $-0.069_{-11}^{+11}$ & - & $88 / 76$ \\
\hline
\end{tabular}

our earlier comments above regarding the probable $\mathcal{O}(a)$ systematics when the $\sigma$ scale was used.

Quantity used to set lattice spacing. In the Adelaide approach, setting the scale from $r_{0}$ gives a significantly smaller $\chi^{2}$ than using $\sigma$ (see figure 11). Given this, and the comments above regarding the probable $\mathcal{O}(a)$ systematics in the $\sigma$ data, we use $r_{0}$ as our preferred method for setting the scale. In the naive case the data do not favour setting the scale from either $r_{0}$ or $\sigma$.

On the basis of these results, we choose the quadratic chiral extrapolation method with the scale set from $r_{0}$ and $\mathcal{O}\left(a^{2}\right)$ corrections in the $a_{0}$ coefficient to define the central value of both the Adelaide and naive fitting procedure. The spread from the other fitting types is used to define the error. In section 5 , we determine the predictions for physical $\rho$-meson mass from these fitting types.

\section{Physical predictions}

We are now ready to estimate $M_{\rho}$ in the continuum using the Adelaide and naive fits performed in the previous section. We obtain this prediction from equations (13) and (14) by setting $M_{\mathrm{PS}}^{\mathrm{deg}}=M_{\mathrm{PS}}^{\text {non-deg }}=M_{\mathrm{PS}}^{\text {unit }}=\mu_{\pi}$. We set $\Delta M_{\omega \rho}$ to zero in this calculation and also note that 
Table 6. Estimates of $\mathrm{M}_{\rho}$ obtained from the global fits. The Adelaide fits used $\Lambda=$ $650(550) \mathrm{MeV}$ when the scale is set from $r_{0}(\sigma)$.

\begin{tabular}{|c|c|c|c|}
\hline Source & Fit procedure & Scale from & $\mathrm{M}_{\rho}(\mathrm{GeV})$ \\
\hline \multirow[t]{2}{*}{ Experiment } & & & 0.770 \\
\hline & \multicolumn{3}{|c|}{ Cubic chiral extrapolation; $a_{0}$ contains $\mathcal{O}\left(a+a^{2}\right)$} \\
\hline \multirow[t]{5}{*}{ Dynamical } & Adelaide & $r_{0}$ & $0.792_{-16}^{+12}$ \\
\hline & Adelaide & $\sigma$ & $0.810_{-11}^{+9}$ \\
\hline & Naive & $r_{0}$ & $0.829_{-16}^{+12}$ \\
\hline & Naive & $\sigma$ & $0.815_{-12}^{+9}$ \\
\hline & \multicolumn{3}{|c|}{ Cubic chiral extrapolation; $a_{0}$ contains $\mathcal{O}\left(a^{2}\right)$ only } \\
\hline \multirow[t]{5}{*}{ Dynamical } & Adelaide & $r_{0}$ & $0.782_{-9}^{+7}$ \\
\hline & Adelaide & $\sigma$ & $0.781_{-7}^{+6}$ \\
\hline & Naive & $r_{0}$ & $0.817_{-9}^{+7}$ \\
\hline & Naive & $\sigma$ & $0.786_{-7}^{+6}$ \\
\hline & \multicolumn{3}{|c|}{ Quadratic chiral extrapolation; $a_{0}$ contains $\mathcal{O}\left(a+a^{2}\right)$} \\
\hline \multirow[t]{5}{*}{ Dynamical } & Adelaide & $r_{0}$ & $0.789_{-13}^{+11}$ \\
\hline & Adelaide & $\sigma$ & $0.805_{-9}^{+8}$ \\
\hline & Naive & $r_{0}$ & $0.837_{-13}^{+11}$ \\
\hline & Naive & $\sigma$ & $0.822_{-9}^{+8}$ \\
\hline & \multicolumn{3}{|c|}{ Quadratic chiral extrapolation; $a_{0}$ contains $\mathcal{O}\left(a^{2}\right)$ only } \\
\hline \multirow[t]{4}{*}{ Dynamical } & Adelaide & $r_{0}$ & $0.779_{-4}^{+4}$ \\
\hline & Adelaide & $\sigma$ & $0.774_{-3}^{+3}$ \\
\hline & Naive & $r_{0}$ & $0.825_{-4}^{+4}$ \\
\hline & Naive & $\sigma$ & $0.791_{-3}^{+3}$ \\
\hline
\end{tabular}

$\Sigma_{\eta^{\prime} \rho}^{\rho}$ vanishes, as required. Since we are predicting the continuum value for the vector meson mass, we calculate the integrals in equations (7) and (8) directly, rather than using the lattice interpretation of the integral in equation (11).

We obtain continuum estimates of all $2^{4}$ fitting types (see table 4 ) using the coefficients, $a_{0}^{\text {cont }}$ and $a_{2,4,6}$, of the global fits of section 4.3 (i.e., those in table 5). Table 6 displays these mass predictions. For the Adelaide case, we use $\Lambda=650(550) \mathrm{MeV}$, with the physical scale set using $r_{0}(\sigma)$ (see section 4.3). Note that we use the global analysis (section 4.3), rather than the analysis of section 4.2 , which treated the ensembles separately, since the global fit is much more tightly constrained.

It is interesting to study the variation in the prediction of the physical mass of the $\rho$ with $\Lambda$. Figure 12 shows how the $M_{\rho}$ prediction varies with $\Lambda$ for each of the Adelaide fits based on the $r_{0}$ scale. Using the $\chi^{2}$ plot in figure 11, we can estimate the range of acceptable $\Lambda$ values defined by increasing $\chi^{2}$ by unity from its minimum, which represents one standard deviation. The horizontal dashed line in figure 11 lies along $\chi^{2}$ values one more than the minimum for the $r_{0}$ case. From this, we determine that the acceptable range of $\Lambda$ values is $620 \leqslant \Lambda \leqslant 690 \mathrm{MeV}$ (see the inset graph in figure 11). This range of $\Lambda$ values is depicted by the vertical dashed lines in figure 12 . 


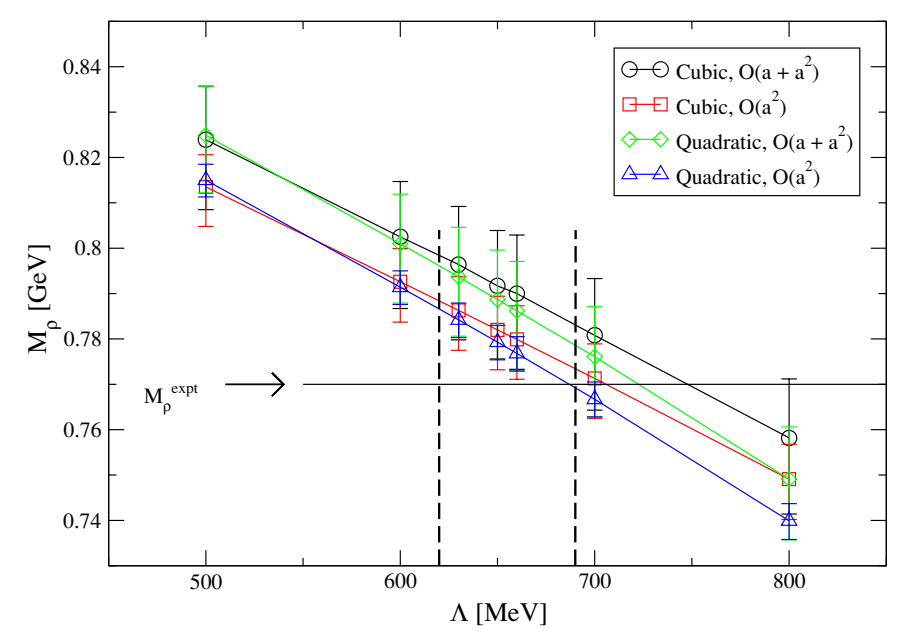

Figure 12. Variation of the physical mass of the $\rho$-meson, $M_{\rho}$, as a function of $\Lambda$, using the Adelaide approach with the scale set from $r_{0}$. The two vertical dashed lines define the range of acceptable $\Lambda$ values $(620 \mathrm{MeV} \leqslant \Lambda \leqslant 690 \mathrm{MeV})$ obtained by increasing $\chi^{2}$ by unity in figure 11 .

From a detailed examination of table 6 and figure 12 we draw the following conclusions:

- The (statistical) errors in the mass estimates are typically around $1 \%$.

- The Adelaide fitting procedure is very stable when the scale is set using $r_{0}$. When the scale is taken from the string tension, the four Adelaide fits are not in mutual agreement. The probable reason for this, as outlined in section 4.3, is that using $\sigma$ to set the scale introduces significant $\mathcal{O}(a)$ errors.

- The Adelaide fitting procedure is quite accurate, lying at most at twice the statistical standard error from the experimental value for the $r_{0}$ case. It would require an uncertainty of only around $1-2 \%$ in $r_{0}$ (and around $2-6 \%$ in $\sqrt{\sigma}$ ) for the Adelaide central values to be in agreement with the experimental value of $M_{\rho}$.

- From figure 12 , the variation of $M_{\rho}$ with $\Lambda$ is small, roughly the same order as the other uncertainties.

- The naive fitting procedure has both a larger spread of values and is further from the experimental value than the Adelaide procedure.

As a result, we conclude that the Adelaide procedure represents a significant improvement over the naive approach.

We obtain final estimates of $M_{\rho}$ by taking the quadratic chiral extrapolation, the scale set from $r_{0}$, and $\mathcal{O}\left(a^{2}\right)$ corrections in the $a_{0}$ coefficient in both the Adelaide and naive fitting procedure. (The reason for this choice of fit type is described in detail in section 4.3.) The central value in the Adelaide case was obtained at $\Lambda=655 \mathrm{MeV}$ from figure 12 (which is an adjustment of $1 \mathrm{MeV}$ from the value obtained at $\Lambda=650 \mathrm{MeV}$ in table 6). The value of $\Lambda=$ $655 \mathrm{MeV}$ was used since it is where the minimum of $\chi^{2}$ occurs in figure 11 . We obtain an estimate of the error in the fit procedure from the spread in the mass predictions using $r_{0}$ for the scale (since we have reservations about the method when the string tension is used to set the scale). An estimate of the uncertainty associated with the determination of $\Lambda$ comes from varying $\chi^{2}$ by unity as described above, i.e., by reading off this error from the vertical dashed lines in figure 12 . Finally then we are led to the following result for the physical mass of the $\rho$-meson: 


$$
\begin{aligned}
& M_{\rho}^{\text {Adelaide }}=778(4)_{-6-9}^{+16+8} \mathrm{MeV}, \\
& M_{\rho}^{\text {Naive }}=825(4)_{-8}^{+12} \mathrm{MeV},
\end{aligned}
$$

where the first error is statistical, the second is from the fit procedure, and, in the Adelaide case, the third error is from the determination of $\Lambda$. The second error on the Adelaide result also includes an uncertainty from the choice of finite-range regulator, which contributes $+3 /-6 \mathrm{MeV}$ to the error [25]. Note that we have not included an error from the determination of $r_{0}$ itself. The only other effect which separates our analysis from nature is that the data we are analysing contain only 2 rather than $2+1$ light dynamical flavours. We have no way to estimate the residual systematic error from this source once $r_{0}$ is matched to the physical value.

The fit parameters shown in table 5 allow one to shift each of the simulation results to the infinite-volume, continuum limit and to remove the effects of partial quenching, hence restoring unitarity in the quark masses. This can be achieved by first considering the preferred form of equation (16), where $X_{1}=a_{6}=0$,

$$
\sqrt{\left(M_{V}^{\mathrm{deg}}\right)^{2}-\Sigma_{\mathrm{TOT}}}=\left(a_{0}^{\mathrm{cont}}+X_{2} a^{2}\right)+a_{2}\left(M_{\mathrm{PS}}^{\mathrm{deg}}\right)^{2}+a_{4}\left(M_{\mathrm{PS}}^{\mathrm{deg}}\right)^{4} .
$$

This can then be used to let $M_{V}^{\mathrm{deg}} \rightarrow M_{V}^{\text {unit }}$ at the same time as removing volume and discretization artefacts. Rewriting equation (20) in terms of the fit vector meson mass gives

$M_{V}^{\mathrm{deg}}(a)=\left[\left(\left(a_{0}^{\mathrm{cont}}+X_{2} a^{2}\right)+a_{2}\left(M_{\mathrm{PS}}^{\mathrm{deg}}\right)^{2}+a_{4}\left(M_{\mathrm{PS}}^{\mathrm{deg}}\right)^{4}\right)^{2}+\Sigma_{\mathrm{TOT}}\left(M_{\mathrm{PS}}^{\mathrm{deg}} ; L\right)\right]^{1 / 2}$.

In the physical continuum limit, this becomes

$M_{V}^{\text {unit }}(a \rightarrow 0)=\left[\left(a_{0}^{\text {cont }}+a_{2}\left(M_{\mathrm{PS}}^{\text {unit }}\right)^{2}+a_{4}\left(M_{\mathrm{PS}}^{\text {unit }}\right)^{4}\right)^{2}+\Sigma_{\mathrm{TOT}}\left(M_{\mathrm{PS}}^{\text {unit }} ; L \rightarrow \infty\right)\right]^{1 / 2}$.

With the unknown parameters determined from the best fit to the entire data set, this then provides a prescription for restoring the physical limit of the data. Specifically, each of the vector meson masses (calculated at finite $a$ and $L$ ) is shifted by an amount

$$
\begin{aligned}
\delta M=\left[\left(a_{0}^{\text {cont }}+\right.\right. & \left.\left.a_{2}\left(M_{\mathrm{PS}}^{\text {unit }}\right)^{2}+a_{4}\left(M_{\mathrm{PS}}^{\text {unit }}\right)^{4}\right)^{2}+\Sigma_{\mathrm{TOT}}\left(M_{\mathrm{PS}}^{\text {unit }} ; L \rightarrow \infty\right)\right]^{1 / 2} \\
& -\left[\left(\left(a_{0}^{\text {cont }}+X_{2} a^{2}\right)+a_{2}\left(M_{\mathrm{PS}}^{\mathrm{deg}}\right)^{2}+a_{4}\left(M_{\mathrm{PS}}^{\mathrm{deg}}\right)^{4}\right)^{2}+\Sigma_{\mathrm{TOT}}\left(M_{\mathrm{PS}}^{\mathrm{deg}} ; L\right)\right]^{1 / 2} .
\end{aligned}
$$

Here it is understood that the pion masses that one should therefore plot against on the $x$-axis are the unitary pion, at the point where the sea mass is held fixed and the valence mass is changed, i.e. $M\left(\beta, \kappa_{\text {sea }} ; \kappa_{\text {val }}, \kappa_{\text {val }}\right) \rightarrow M\left(\beta, \kappa_{\text {sea }} ; \kappa_{\text {sea }}, \kappa_{\text {sea }}\right)$. The final estimate of the physical point is given by

$$
\left[M_{V}^{\text {unit }}(a \rightarrow 0 ; L \rightarrow \infty)\right]_{\text {estimate }}=\left[M_{V}^{\mathrm{deg}}\right]_{\text {lattice }}+\delta M .
$$

The results of these shifts are displayed in figure 13, where we observe a remarkable result. The tremendous spread of data seen in figure 10 is dramatically reduced, with all 80 points now lying very accurately on a universal curve.

One feature of figure 10 is the very steady approach to the chiral limit from the physical point. This yields a value for the vector meson mass in the chiral limit $\left(M_{\rho}^{0}\right)$ that is very near the physical value. To remove the overall scale, we report on the mass shift between the physical and chiral values, finding

$$
M_{\rho}-M_{\rho}^{0}=3.7(2)(4)(8) \mathrm{MeV},
$$

where the sources of uncertainty are the same as those reported above for $M_{\rho}$ in equation (18). This small number indicates that the sigma term for the $\rho$ is significantly smaller than the nucleon, where the derivative is observed to increase near the chiral limit [2]. The reduced slope in the case of the $\rho$ arises from the presence of substantial spectral strength in the low-energy two-pion channel below the $\rho$-meson mass [26]. 


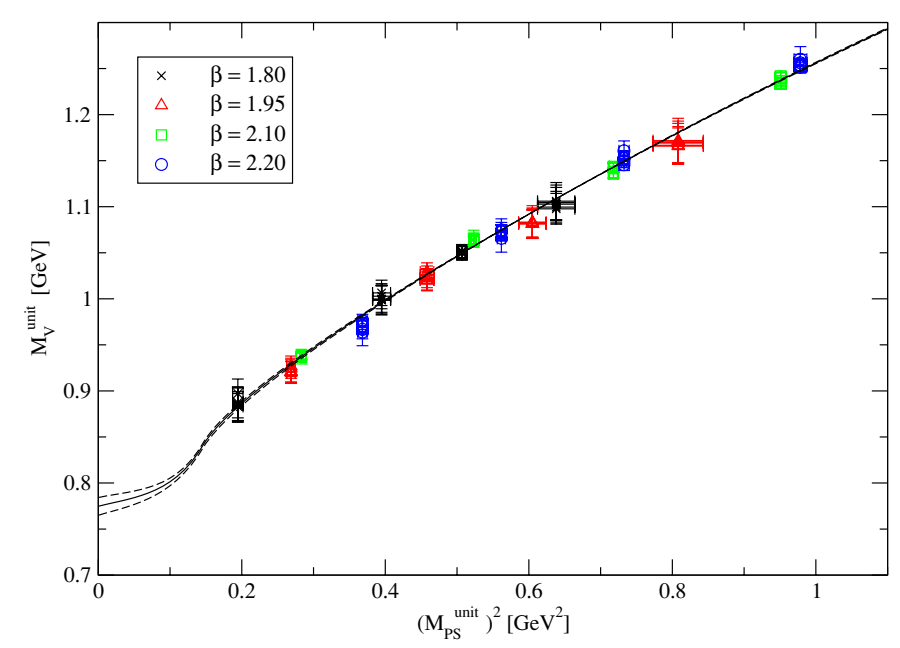

Figure 13. The same 80 lattice data points as in figure 10, after correction to restore the infinitevolume, continuum and quark-mass unitarity limits. The central curve displays the best-fit from the global analysis with $\Lambda=655 \mathrm{MeV}$. The dashed curves show the (statistically constrained) bounds on the FRR scale, $0.620<\Lambda<0.690 \mathrm{GeV}$.

\section{Conclusions}

In summary, we have tackled the ambitious task of producing a single, unified chiral fit to all of the accurate CP-PACS data for the mass of the $\rho$-meson in partially quenched QCD, i.e., the case where $\kappa_{\text {sea }} \neq \kappa_{\text {val }}$. As well as using a naive polynomial fit in $M_{\mathrm{PS}}^{2}$, we have generalized the Adelaide approach, developing a unified analysis approach, to fit the data. This approach enables one to account for finite volume errors by evaluating the chiral selfenergy contributions with the same momentum discretization implicit in the lattice simulations. In addition, we have been able to quantify the residual $\mathcal{O}(a)$ effects and hence carry out a continuum extrapolation (cf figures 8 and 9).

The $\chi^{2}$ obtained for the Adelaide fit, with the physical scale set using $r_{0}$, is a factor of two lower than that for any other method. This provides considerable confidence in the method, even before it is used to produce the physical mass of the $\rho$. The quality of the fit leads to very small (statistical) error bars (see table 6).

In addition, it is possible to estimate the systematic errors in the extrapolation to the physical $\rho$-mass associated with the fitting procedure (both the chiral and continuum-limit fitting procedures). In particular, the finite-range regulator parameter, $\Lambda$, is constrained by the model-independent lattice QCD data, and the variation of the $M_{\rho}$ prediction within this range was found to be $1 \%$.

The curve through figure 13 displays the determined variation of the $\rho$-meson mass with pion mass. This curve also presents an extrapolation to the physical point, allowing extraction of the physical $\rho$-meson mass

$$
M_{\rho}=778(4)_{-6-9}^{+16+8} \mathrm{MeV},
$$

where the first error is statistical, the second is from variations of the fit procedure and the third from the determination of $\Lambda$. Whereas the naive fitting procedure leads to a value that is $50-60 \mathrm{MeV}$ too high, the result from the chiral analysis is in excellent agreement with the experimentally observed mass. 


\section{Acknowledgments}

CRA and WA would like to thank the CSSM for their support and kind hospitality. WA would like to thank PPARC for travel support. The authors would like to thank Stewart Wright and Graham Shore for helpful comments. This work was supported by the Australian Research Council and by DOE contract DE-AC05-84ER40150, under which SURA operates Jefferson Laboratory.

\section{References}

[1] Ali Khan A et al (CP-PACS Collaboration) 2002 Phys. Rev. D 65054505 (Preprint hep-lat/0105015) Ali Khan A et al (CP-PACS Collaboration) 2003 Phys. Rev. D 67059901 (erratum)

[2] Leinweber D B, Thomas A W and Young R D 2004 Phys. Rev. Lett. 92242002 (Preprint hep-lat/0302020)

[3] Procura M, Hemmert T R and Weise W 2004 Phys. Rev. D 69034505 (Preprint hep-lat/0309020)

[4] Leinweber D B, Thomas A W, Tsushima K and Wright S V 2000 Phys. Rev. D 61074502 (Preprint hep-lat/9906027)

[5] Durr S 2003 Eur. Phys. J. C 29383 (Preprint hep-lat/0208051)

[6] Bernard C, Hashimoto S, Leinweber D B, Lepage P, Pallante E, Sharpe S R and Wittig H 2003 Nucl. Phys. Proc. Suppl. 119170 (Preprint hep-lat/0209086)

[7] Young R D, Leinweber D B and Thomas A W 2003 Prog. Part. Nucl. Phys. 50399 (Preprint hep-lat/0212031)

[8] Beane S R 2004 Nucl. Phys. B 695192 (Preprint hep-lat/0403030)

[9] Thomas A W, Guichon P A M, Leinweber D B and Young R D 2004 Prog. Theor. Phys. Suppl. 156124 (Preprint nucl-th/0411014)

[10] Leinweber D B, Thomas A W and Young R D 2005 Nucl. Phys. A 75559 (Preprint hep-lat/0501028)

[11] Donoghue J F, Holstein B R and Borasoy B 1999 Phys. Rev. D 59036002 (Preprint hep-ph/9804281)

[12] Djukanovic D, Schindler M R, Gegelia J and Scherer S 2005 Phys. Rev. D 72045002

[13] Young R D, Leinweber D B and Thomas A W 2005 Phys. Rev. D 71014001 (Preprint hep-lat/0406001)

[14] Golterman M F L and Leung K C L 1998 Phys. Rev. D 575703 (Preprint hep-lat/9711033)

[15] Sharpe S R and Shoresh N 2001 Phys. Rev. D 64114510 (Preprint hep-lat/0108003)

[16] Chen J W and Savage M J 2002 Phys. Rev. D 65094001 (Preprint hep-lat/0111050)

[17] Beane S R and Savage M J 2002 Nucl. Phys. A 709319 (Preprint hep-lat/0203003)

[18] Leinweber D B 2004 Phys. Rev. D 69014005 (Preprint hep-lat/0211017)

[19] Arndt D and Tiburzi B C 2003 Phys. Rev. D 68094501 (Preprint hep-lat/0307003)

[20] Arndt D and Lin C-J D 2004 Phys. Rev. D 70014503 (Preprint hep-lat/0403012)

[21] Bijnens J, Danielsson N and Lahde T A 2004 Phys. Rev. D 70111503 (Preprint hep-lat/0406017)

[22] Detmold W and Lin C-J D 2005 Phys. Rev. D 71054510 (Preprint hep-lat/0501007)

[23] Bijnens J, Gosdzinsky P and Talavera P 1997 Nucl. Phys. B 501495 (Preprint hep-ph/9704212)

[24] Bruns P C and Meissner U G 2005 Eur. Phys. J. C 4097 (Preprint hep-ph/0411223)

[25] Allton C R, Armour W, Leinweber D B, Thomas A W and Young R D 2005 Phys. Lett. B 628125 (Preprint hep-lat/0504022)

[26] Leinweber D B and Cohen T D 1994 Phys. Rev. D 493512 (Preprint hep-ph/9307261)

[27] Leinweber D B, Thomas A W, Tsushima K and Wright S V 2001 Phys. Rev. D 64094502 (Preprint hep-lat/0104013)

[28] Labrenz J N and Sharpe S R 1996 Phys. Rev. D 544595 (Preprint hep-lat/9605034)

[29] Chow C K and Rey S J 1998 Nucl. Phys. B 528303 (Preprint hep-ph/9708432)

[30] Young R D, Leinweber D B, Thomas A W and Wright S V 2002 Phys. Rev. D 66094507 (Preprint hep-lat/0205017)

[31] Sommer R 1994 Nucl. Phys. B 411839 (Preprint hep-lat/9310022)

Edwards R G, Heller U M and Klassen T R 1998 Nucl. Phys. B 517377 (Preprint hep-lat/9711003)

[32] Edwards R G, Heller U M and Klassen T R 1998 Phys. Rev. Lett. 803448 (Preprint hep-lat/9711052) Zanotti J M, Lasscock B, Leinweber D B and Williams A G 2005 Phys. Rev. D 71034510 (Preprint hep-lat/0405015)

[33] Bär O 2005 Nucl. Phys. Proc. Suppl. 140106 (Preprint hep-lat/0409123)

[34] Rupak G and Shoresh N 2002 Phys. Rev. D 66054503 (Preprint hep-lat/0201019)

[35] Bar O, Rupak G and Shoresh N 2004 Phys. Rev. D 70034508 (Preprint hep-lat/0306021)

[36] Aoki S 2003 Phys. Rev. D 68054508 (Preprint hep-lat/0306027)

[37] Beane S R and Savage M J 2003 Phys. Rev. D 68114502 (Preprint hep-lat/0306036)

[38] Grigoryan H R and Thomas A W 2006 Phys. Lett. B 632657 (Preprint hep-lat/0507028) 